\title{
Study on the Rectification of Forebay in Pumping Station
}

\author{
Weiwei Song $\left(\mathbb{D},{ }^{1}\right.$ Yong Pang, ${ }^{2}$ Xiaohuan Shi, ${ }^{3}$ and Qing $\mathrm{Xu}^{4}$ \\ ${ }^{1}$ College of Hydrology and Water Resources, Hohai University, Nanjing 210098, China \\ ${ }^{2}$ College of Environment, Hohai University, Nanjing 210098, China \\ ${ }^{3}$ Shanghai YouWei Engineering Design Co., Ltd., Shanghai 200333, China \\ ${ }^{4}$ School of Hydraulic, Energy and Power Engineering, Yangzhou University, Yangzhou 225009, China \\ Correspondence should be addressed to Weiwei Song; weiweisong@hhu.edu.cn
}

Received 19 August 2017; Revised 21 December 2017; Accepted 8 January 2018; Published 4 March 2018

Academic Editor: Giuseppe Vairo

Copyright (C) 2018 Weiwei Song et al. This is an open access article distributed under the Creative Commons Attribution License, which permits unrestricted use, distribution, and reproduction in any medium, provided the original work is properly cited.

The forebay of pumping stations is an important hydraulic structure that connects the channel with the inlet channel. Actual test observations and theoretical studies have shown that poor precursors produce backflow, vortex, and water flow disturbances in the forebay water. In this paper, taking a lateral inlet pump station as an example, we study the nonmeasures and five rectification measures- "Y" type diversion pier, "T" shaped diversion pier, narrow bottom hole, high and wide bottom, and diversion wall - through adopting the method of numerical simulation and model test. For the numerical simulation, the corresponding three-dimensional model is established by UG solid modeling software, and then the computational fluid is simulated numerically with CFX. Based on the analysis and comparison of the results during the test of numerical simulation and model test, the stability of the rectification measures is considered after taking into consideration the results of the uniformity test of the velocity distribution of the surface layer, the bottom layer, and the front section of each scheme. The proposed scheme 3 ("T" diversion pier) is regarded as the pumping station flow control measures.

\section{Introduction}

To operate the pump safely, the water flow in the foreland is a very important factor. It is very important for the pump station to be stable and safe to operate. In many pumping stations during operation, many phenomena are related to poor water flow, such as severe vibration, low efficiency, and serious cavitation. We studied the influencing factors of the flow regime of the forebay and the causes of the bad flow. After that, the corresponding rectification measures were put forward, and various engineering measures of the geometric parameters were adjusted to change its influence on the improvement of the flow regime. Setting the bottom and the columns in the forebay can improve the flow, and then we can prepare the brewing in the pool before the whirlpool and swing and other bad flow [1]. The characteristics of lateral flow regime and flow characteristics and the principle of rectification are described in detail, and several flow optimization measures used commonly and research methods are also analyzed in detail [2]. Based on the hydraulic model test of a large-scale urban water source pumping station, the eight-shaped diversion pier, non-full-section bottom grill, and vanishing plate are proposed to improve the influent flow regime [3]. The uniformity and stability of the fluid in the forebay are studied by the size, strength, uniformity of the velocity, and the form of water connection [4]. Combined with the practice in engineering, we made a conclusion about a series of measures to optimize the flow pattern of the forebay, including selecting the bottom and the column, adding the pier, and placing the water pressure plate and the diversion pier. Analyzing and comparing all kinds of optimized measures, we will expound the advantages and disadvantages of various schemes in detail [5].

CFD technology has become mature, and numerical simulation for pumping stations has also emerged, so the flow of the former pool and its impact were predicted and evaluated through using numerical simulation technology. Adopting the method of the Fluent software to analyze the geometrical parameters of the $Y$ shaped diversion piers and analyzing the rectification effect, it is shown that the $\mathrm{Y}$ shaped diversion 
TABLE 1: Scale for physical model test and hydraulic volume conversion ratio.

\begin{tabular}{lcc}
\hline $\begin{array}{l}\text { Hydraulic conversion } \\
\text { ratio }\end{array}$ & Conversion formula & Model scale \\
\hline $\begin{array}{l}\text { Horizontal length } \\
\text { scale }\end{array}$ & $X_{\gamma}$ & 25 \\
Vertical length scale & $Y_{\gamma}$ & 25 \\
Area ratio & $X_{\gamma} Y_{\gamma}$ & 625 \\
Velocity ratio & $Y_{\gamma}^{1 / 2}$ & 5 \\
Flow ratio & $X_{\gamma} Y_{\gamma}^{3 / 2}$ & 3125 \\
Slope ratio & $Y_{\gamma} X_{\gamma}$ & 625 \\
$\begin{array}{l}\text { Roughness coefficient } \\
\text { ratio }\end{array}$ & $X_{\gamma}^{-1 / 2} Y_{\gamma}^{2 / 3}$ & 0.585 \\
\hline
\end{tabular}

piers have a good effect on the whole dispersion water flow. At the same time, the diversion of the tail will appear after the whirlpool, adjusting the $\mathrm{Y}$ shaped diversion pier position, height, angle, and length to adjust the flow [6]. Through using the realizable turbulence model and the N-S equation, the numerical simulation is carried out for the forward water front without the optimization measures. The results of numerical simulation show that it is important to set the appropriate position and the high value of the bottom hole to achieve the optimal rectification effect [7]. Fluent software was used to simulate the three turbulence models used in common. At the same time, we compared and analyzed the results of the model test and the influence of the arrangement, size, and number of the baffles and the bottom [8]. The SIMPLE algorithm is used to simulate the flow velocity and velocity vector diagram of the forebay water flow and simulate the change of the diffusion angle and the effect of different starting schemes on the influent flow regime [9].

In this paper, at one side of the inlet pump station, if the 4 900ZLB-70 axial flow pump is set up in the pump station, flow will be $10 \mathrm{~m}^{3} / \mathrm{s}$. As for the lateral water of the station, to get a good water flow and ensure a safe economical operation of the pump station, we carried out a study on the station before the pool flow model test and numerical simulation. There are no measures but five rectification measures: "Y" type diversion pier, "T" shaped diversion pier, narrow bottom, high and wide bottom, and diversion wall (Table 2). The import plane layout of the pump station is shown in Figure 1.

\section{Materials and Methods}

2.1. Model Test. In the study of a part of the hydraulic structure of the water flow, the local model can be used; the scale should not be less than 1:50 [10-12]. In this phase of the test, in order to improve the test accuracy, take the model ratio $=25\left(\lambda=X_{\gamma}=Y_{\gamma}\right)$. The scale for the physical model test and hydraulic volume conversion ratio is shown in Table 1.

The flow pattern of the forebay of the pumping station includes the river, the front tank, the inlet, and the inlet pipe (including the horn tube). The overall layout of the flow pattern of the forebay of the pumping station is shown in Figure 2, and a panorama of the pumping station forebay flow model test is shown in Figure 3. The upper boundary adopts the maximum water depth of 6.5 times the inlet of the model river and the downstream boundary of the model river takes the maximum surface width of 1.5 times the inlet of the model river. During the test, the model is supplied by the water supply pump. The flow rate of each pump is measured by four electromagnetic flowmeters in the pipeline. The four pumps are adjusted to ensure the same flow rate. The prototype and the wing wall of the prototype pump station are pulp stone, with a coefficient of roughness of 0.025 . The prepond and inlet channel are reinforced concrete with a roughness coefficient of 0.011 to 0.020 . The river bottom does not protect the puzzle; the roughness coefficient is 0.0225 . Therefore, the roughness of the model material needs to be in the range from 0.0064 to 0.0146 . The model materials used are a Plexiglass plate and a plastic plate, with the roughness coefficients of 0.007 and 0.011 , respectively, which basically meet the requirements of similar roughness.

\subsection{Numerical Simulation}

2.2.1. Numerical Simulation Theory. During the operation of pumping, the flow of water in the front tank can be regarded as a complex three-dimensional incompressible turbulent flow. The unsteady continuous equations and the instantaneous Navier-Stokes equations are suitable for turbulence applications [13-15]. In this paper, a RNG $k-\varepsilon$ turbulence model is established from the three-dimensional incompressible Navier-Stokes equations [16-21].

(1) Control Equations. The fluid flow in the forebay of the pumping station satisfies the law of conservation of momentum:

$$
\frac{\partial u_{i}}{\partial x_{i}}=0
$$

where $u_{i}$ is the velocity component along the direction of $i$ and $x_{i}(i=1,2,3)$ is the coordinate axis:

$$
u_{j} \frac{\partial u_{i}}{\partial x_{j}}=g-\frac{1}{\rho} \frac{\partial p}{\partial x_{i}}+\frac{\partial}{\partial x_{j}}\left[\left(v+v_{i}\right)\left(\frac{\partial u_{i}}{\partial x_{j}}+\frac{\partial u_{j}}{\partial x_{i}}\right)\right],
$$

where $p$ is the pressure, $\rho$ is the density, $g$ is the gravitational acceleration, $v$ is the kinematic viscosity coefficient of water, and $v_{i}$ is the turbulence viscosity coefficient.

(2) Turbulence Model. The RNG $k-\varepsilon$ model takes into account the rotation and swirl in the average flow, which can better handle the high strain rate and flow with a greater degree of flow line curvature. Orszag and Yakhot built the RNG $k-\varepsilon$ model for the first time and demonstrated its superiority over the traditional method [13], and others have made good use of this model $[14,15]$.

Continuous equation:

$$
\frac{\partial \rho}{\partial t}+\frac{\partial\left(\rho u_{j}\right)}{\partial x_{j}}=0
$$




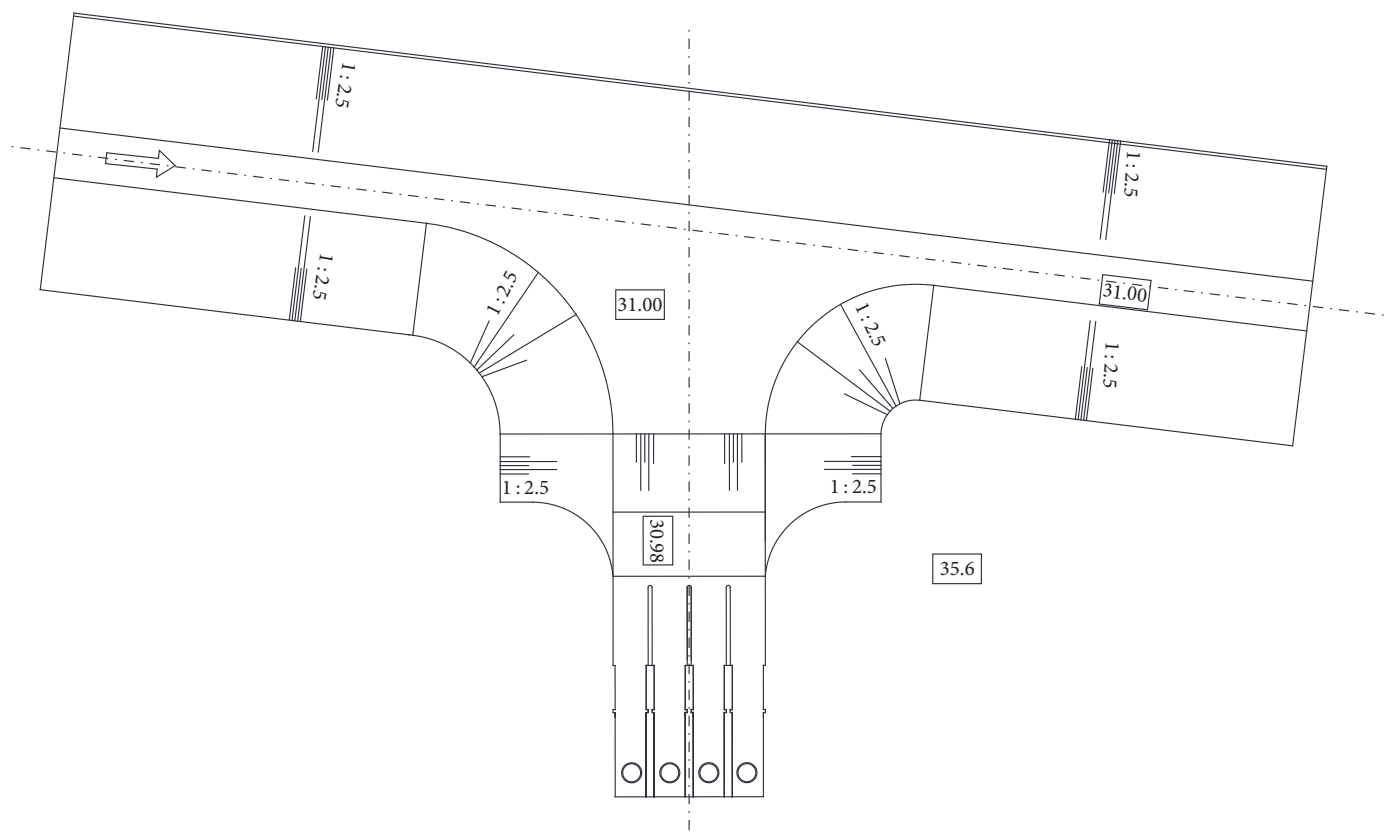

FIGURE 1: Layout plan of the pump station.

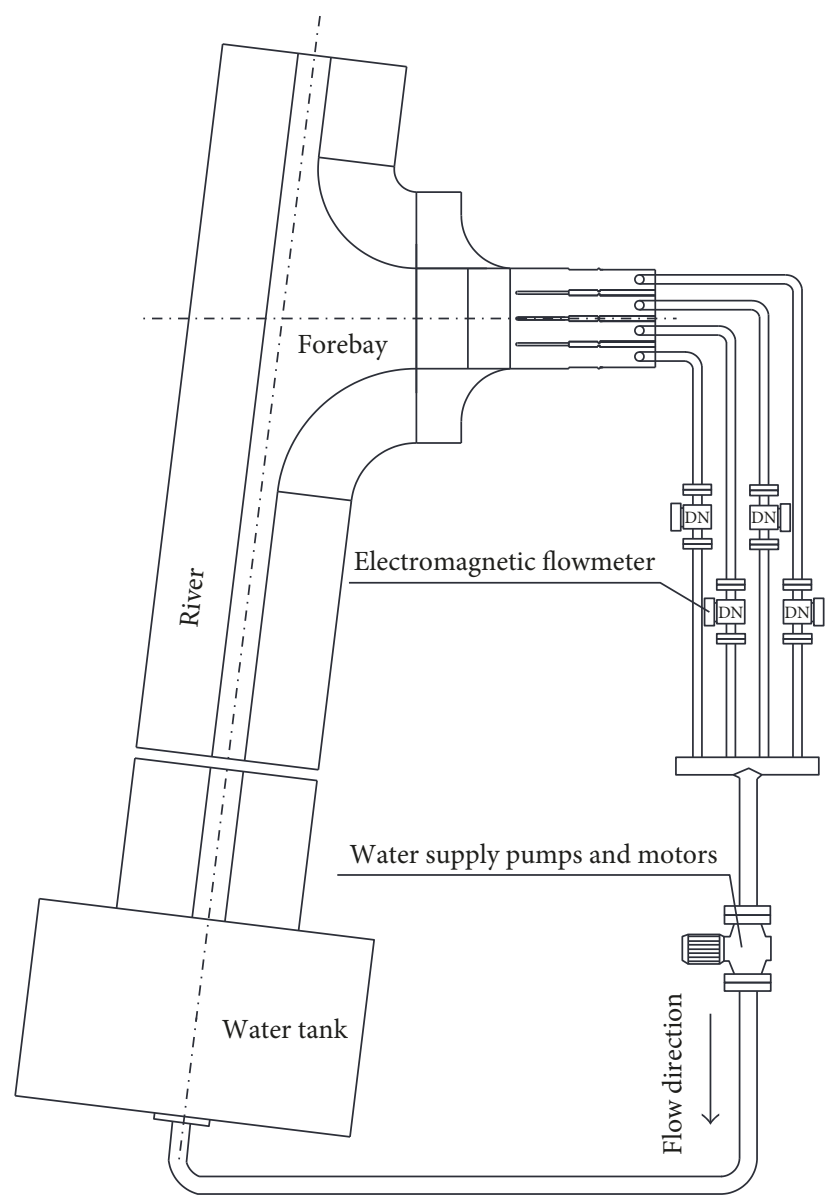

FIGURE 2: Overall layout of the flow pattern of the forebay of the pumping station. 
TABLE 2: List of test schemes for flow pattern of pumping station.

\begin{tabular}{|c|c|c|}
\hline Program number & Plan description & Action description (prototype in parentheses) \\
\hline 1 & Original plan & Without any action (Figure 2). \\
\hline 2 & "Y" type diversion pier & $\begin{array}{l}\text { The "Y" type diversion pier top elevation is equal } \\
\text { to the lowest water level. Side length: } 10 \mathrm{~cm} \\
(2.5 \mathrm{~m}) \text { (Figure } 4) .\end{array}$ \\
\hline 3 & " $\mathrm{T}$ " shaped diversion pier & $\begin{array}{l}\text { The "T" type diversion pier top elevation is equal } \\
\text { to the lowest water level. Side length: } 10 \mathrm{~cm} \\
(2.5 \mathrm{~m}) \text {. The edges of the diversion piers have } \\
\text { changed (Figure 5). }\end{array}$ \\
\hline 4 & $4 \mathrm{~cm}$ narrow bottom & Bottom hole height: $4 \mathrm{~cm}(H=1 \mathrm{~m})$ (Figure 6). \\
\hline 5 & High and wide bottom & $\begin{array}{l}\text { High and wide bottom width of } 6 \mathrm{~cm}(1.5 \mathrm{~m}) \text {; top } \\
\text { width: } 3 \mathrm{~cm}(0.75 \mathrm{~m}) \text {; bucket top elevation: } 6 \mathrm{~cm} \\
(1.5 \mathrm{~m}) \text { (Figure } 7) .\end{array}$ \\
\hline 6 & Diversion wall & $\begin{array}{l}\text { The diversion wall elevation is equal to the } \\
\text { minimum water level. Length: } 30 \mathrm{~cm}(7.5 \mathrm{~m}) \\
\text { (Figure 8). }\end{array}$ \\
\hline
\end{tabular}

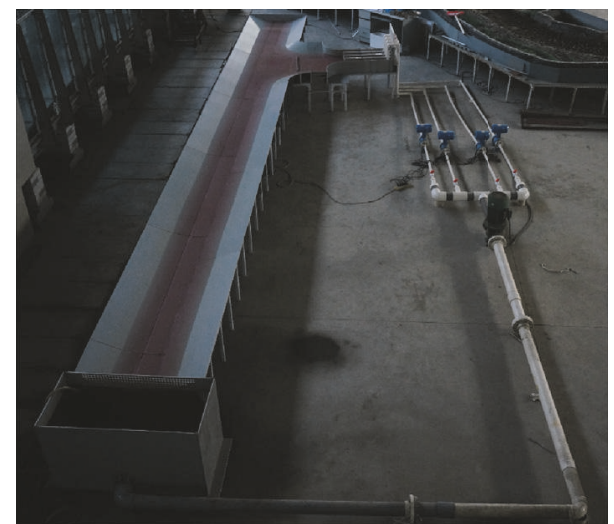

FIGURE 3: Panorama of the pumping station forebay flow model test.

Momentum equation:

$$
\begin{aligned}
\frac{\partial\left(\rho u_{i}\right)}{\partial t}+\frac{\partial\left(\rho u_{j} u_{i}\right)}{\partial x_{j}}= & -\frac{\partial p^{\prime}}{\partial x_{i}} \\
& +\frac{\partial}{\partial x_{j}}\left[\mu_{\mathrm{eff}}\left(\frac{\partial u_{i}}{\partial x_{j}}+\frac{\partial u_{j}}{\partial x_{i}}\right)\right] \\
& +\rho g_{i} .
\end{aligned}
$$

$k$ equation and $\varepsilon$ equation:

$$
\begin{aligned}
\frac{\partial(\rho k)}{\partial t}+\frac{\partial(\rho k) u_{j}}{\partial x_{j}}= & \frac{\partial}{\partial x_{j}}\left(\alpha_{k} \mu_{e} \frac{\partial k}{\partial x_{j}}\right)+\rho\left(P_{k}-\varepsilon\right), \\
\frac{\partial(\rho \varepsilon)}{\partial t}+\frac{\partial\left(\rho \varepsilon u_{j}\right)}{\partial x_{j}}= & \frac{\partial}{\partial x_{j}}\left(\alpha_{\varepsilon} \mu_{e} \frac{\partial \varepsilon}{\partial x_{j}}\right) \\
& +\rho \frac{\varepsilon}{k}\left(C_{1 \varepsilon}^{*} P_{k}-C_{2 \varepsilon} \varepsilon\right),
\end{aligned}
$$

where $C_{1 \varepsilon}^{*}=C_{1 \varepsilon}-\left(\eta\left(1-\eta / \eta_{0}\right)\right) /\left(1+\beta \eta^{3}\right) ; \eta=\left(2 E_{i j} E_{i j}\right)^{1 / 2} k / \varepsilon$; $E_{i j}=(1 / 2)\left(\partial u_{i} / \partial x_{j}+\partial u_{j} / \partial x_{i}\right)$; the empirical coefficient $\alpha_{k}=$ $\alpha_{\varepsilon}=1.39 ; C_{1 \varepsilon}=1.42 ; C_{2 \varepsilon}=1.68 ; \eta_{0}=4.377 ; \beta=0.012$.

The RNG $k-\varepsilon$ model comes from rigorous statistical techniques. The empirical coefficient comes from the physical model. It is similar to the standard $k-\varepsilon$ model, but with the following improvements: (a) the RNG model adds a condition to the $\varepsilon$ equation, which effectively improves the accuracy; (b) taking into account the turbulent eddies improves the accuracy in this regard; (c) the RNG theory provides an analytical formula for turbulent Prandtl numbers, whereas the standard $k-\varepsilon$ model uses user-supplied constants; (d) the standard $k-\varepsilon$ model is a model of high Reynolds number and RNG theory provides an analytical formula that takes into account the low viscous flow of Reynolds numbers. The role of these formulas depends on the correct treatment of the near wall region. These features make the RNG $k-\varepsilon$ model more reliable and accurate than the standard $k-\varepsilon$ model in a wider range of fluid motions.

2.2.2. Model Establishment. The first step in numerical simulation is the modeling of three-dimensional entities. It is an important factor affecting the lattice division and the accuracy of the final research results whether the model is accurate or not [22-27]. The simulation range of the pumping station is $162.5 \mathrm{~m}$ for the upper reaches of the river and $37.5 \mathrm{~m}$ for the lower reaches of the river. Design water level is $34 \mathrm{~m}$. The numerical simulation of the fluid area is shown in Figure 9, and enlarged details of the pump station are shown in Figure 10.

2.2.3. Grid Division and Sensitivity Verification. In this paper, meshes are used to divide the tetrahedron unstructured meshes with the method of RNG $k-\varepsilon$ turbulence model. The calculation area of the calculated fluid consists of a river inlet, a pump outlet, a river side wall, and a free surface. When simulation calculations use design condition, the import boundary condition is the mass flow inlet. The 


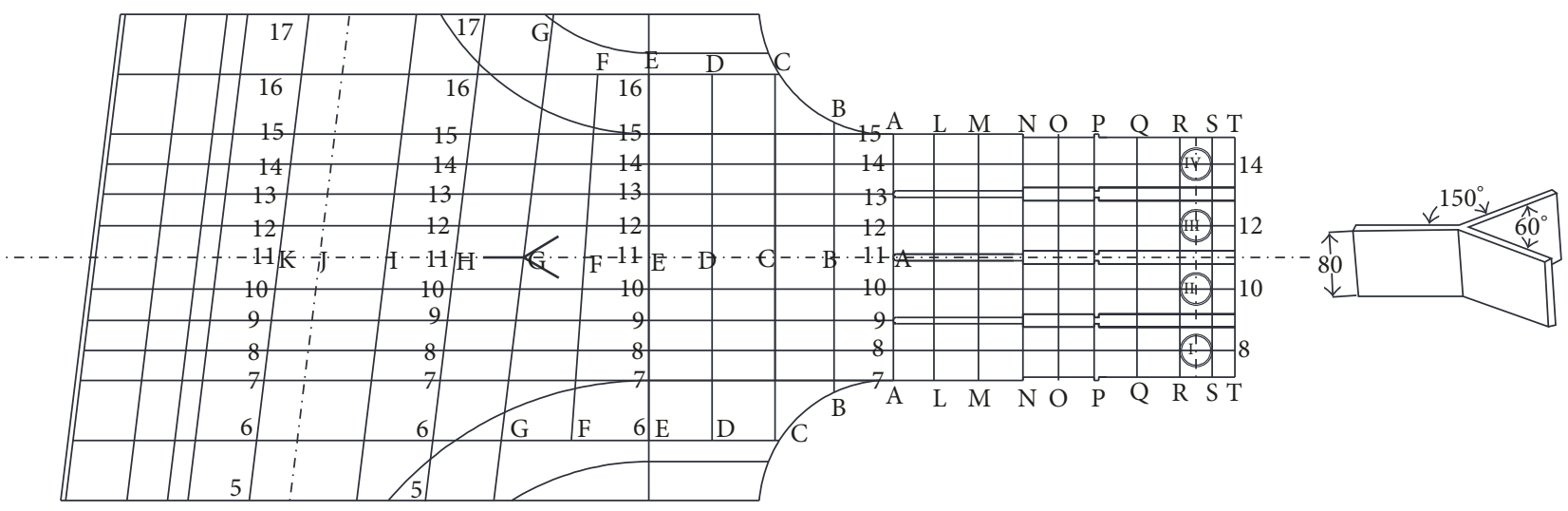

FIGURE 4: "Y" type guide pier position and shape diagram.
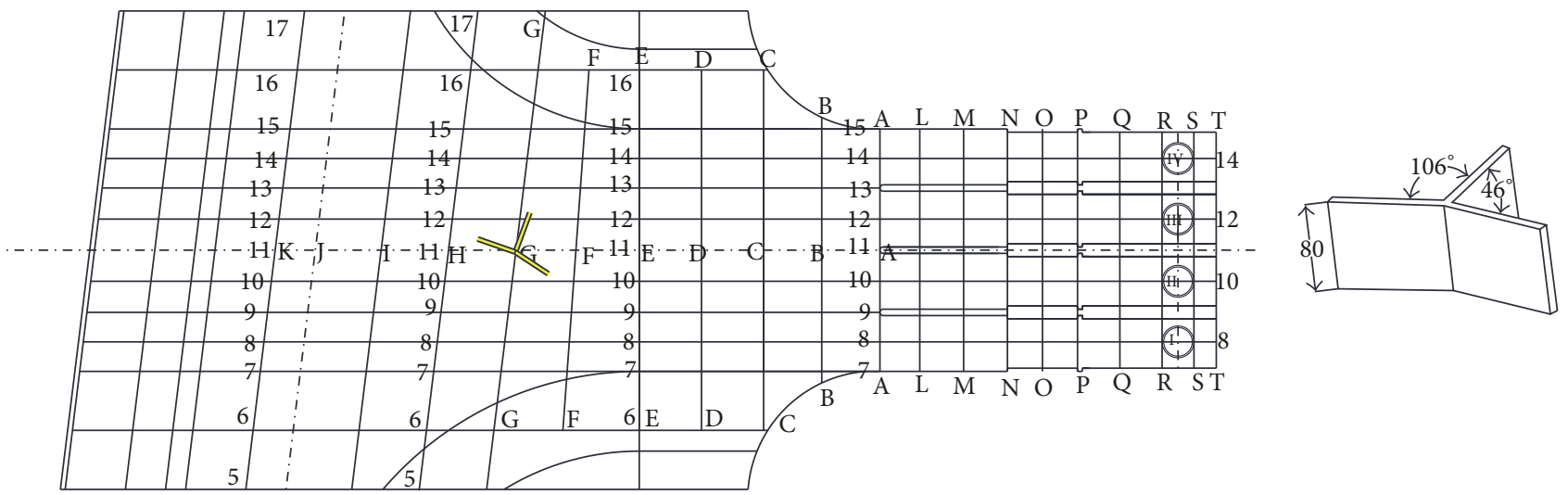

FIgURE 5: "T" type guide pier position and shape diagram.
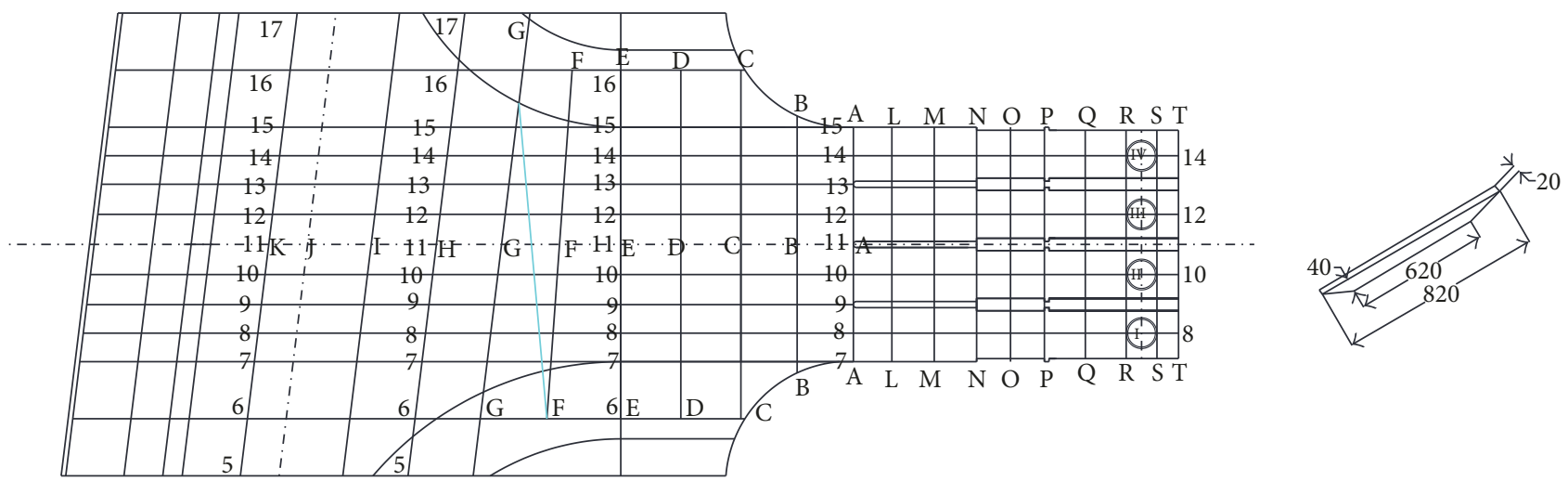

FiguRE 6: Narrow bottom position and shape diagram.

free-water surface adopts the steel cover theorem to use the outflow and uses the symmetrical boundary condition. The export condition is free flowing; the fluid and the wall have no slip [28-32]. The pump station forebay grid map is shown in Figure 11(a). Velocity uniformity under different grid numbers is shown in Table 3.

Mesh sensitivity verification, that is, changing the mesh density several times, after calculating the results, compares the changes in the calculated results. If the variation range is within the allowable error range, it is considered that the grid density has reached the expected accuracy of calculation. It can also be said that the degree of grid density is suitable. In this paper, due to the cross section of the inlet basin for the flow rate uniformity test, when the velocity uniformity changes within a reasonable range, the grid density becomes reasonable. The verification results are shown in Table 3 and Figure 11(b).

When the grid reaches 852241, 950476, and 1196985, the error of the corresponding velocity uniformity becomes $-2.1 \%$ and $-2.9 \%$ under the condition of no rectification 


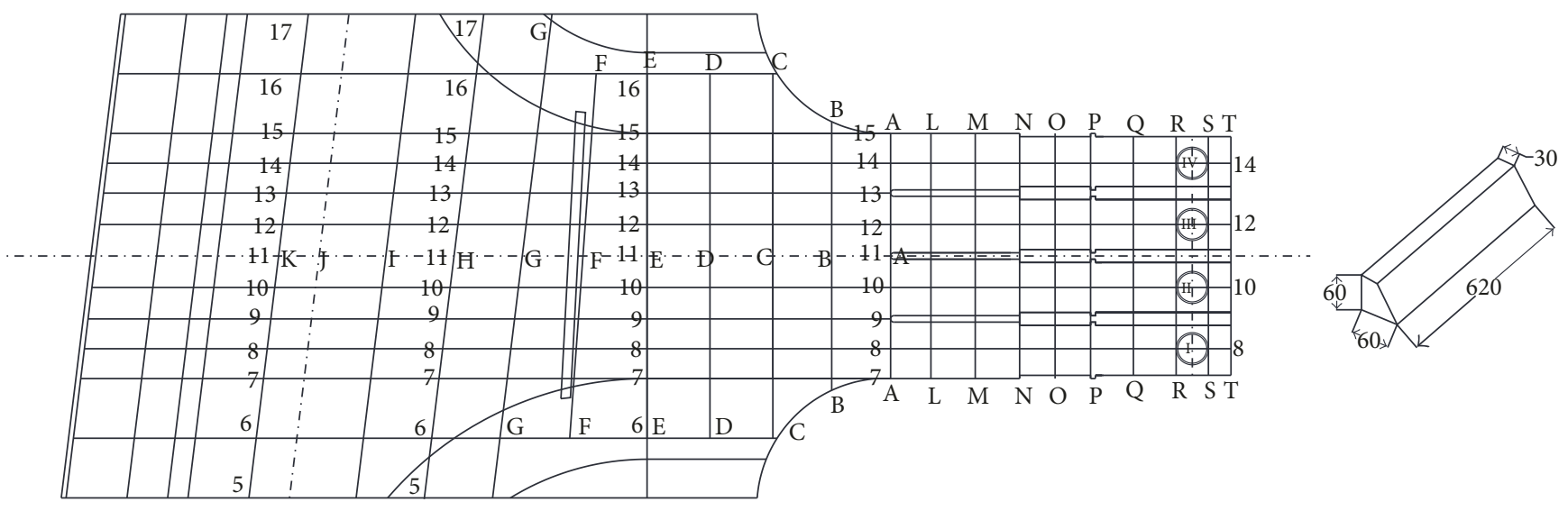

FIgURE 7: High-width bottom position and shape diagram.
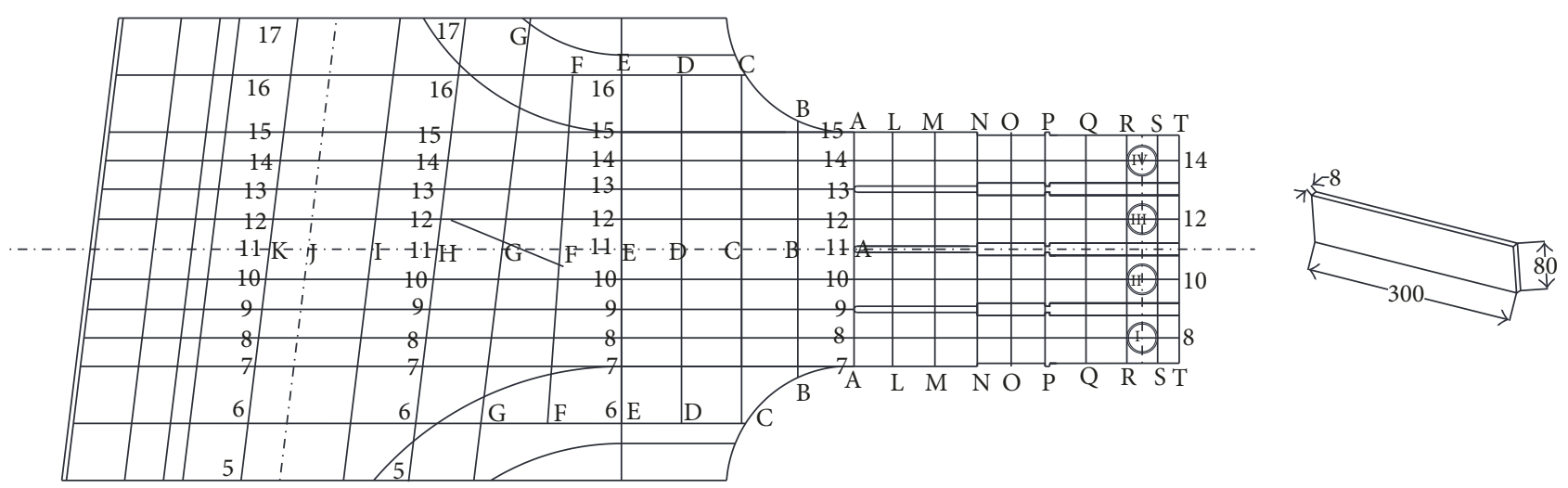

FIGURE 8: Guide wall location and shape diagram.

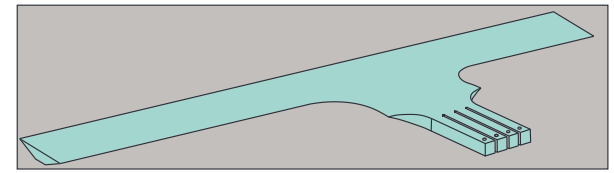

Figure 9: Pump design water level calculation fluid model.

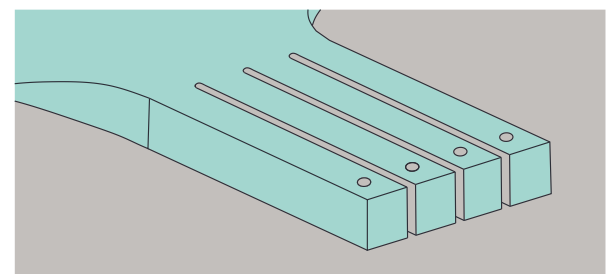

Figure 10: Pump station water intake details.

measures. It can be considered that the calculation result of the grid with the number 950476 is irrelevant to the degree of grid density. Therefore, other models calculated in this paper adopt this grid density.

\section{Results and Discussion}

\subsection{Flow Pattern Comparison of the Model Test and Numerical Simulation}

3.1.1. Error Analysis. The results of numerical simulation and model test are almost impossible to be identical. The analysis errors are as follows.

(1) If the surface of free water is of steel cover and symmetrical boundary conditions, that is to say, the free water is always completely level, the surface layer of flow cannot accurately be described, and steel cover assumptions ignore the impact of air resistance.

(2) With the floating plastic particles, to characterize the surface layer of fluid, the interaction among particles will also affect the conditions of the water surface movement (Figure 12).

(3) The influence of refractive index: in drawing the flow diagram of the surface layer, the center and scope of the vortex are measured by the naked eye. Actually, this way, it is difficult to ensure that the viewing angle has been perpendicular to the surface to locate the whirlpool in the grid line position.

In view of the two cases, these existing errors are basically similar to the flow chart, including the location of the whirlpool and its size. 
TABLE 3: Velocity uniformity under different grid numbers.

\begin{tabular}{lcccccc}
\hline Number of grids & 757355 & 852241 & 950476 & 1196985 & 1285549 & 1070463 \\
Flow rate uniformity \% & 82.54648 & 86.55 & 84.66543 & 82.13911 & 83.20061 & 84.10678 \\
\hline
\end{tabular}

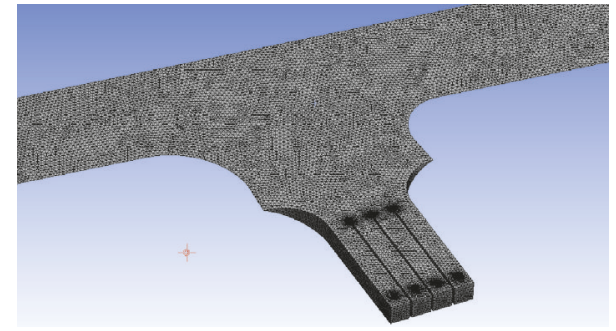

(a) Pump station forebay grid map

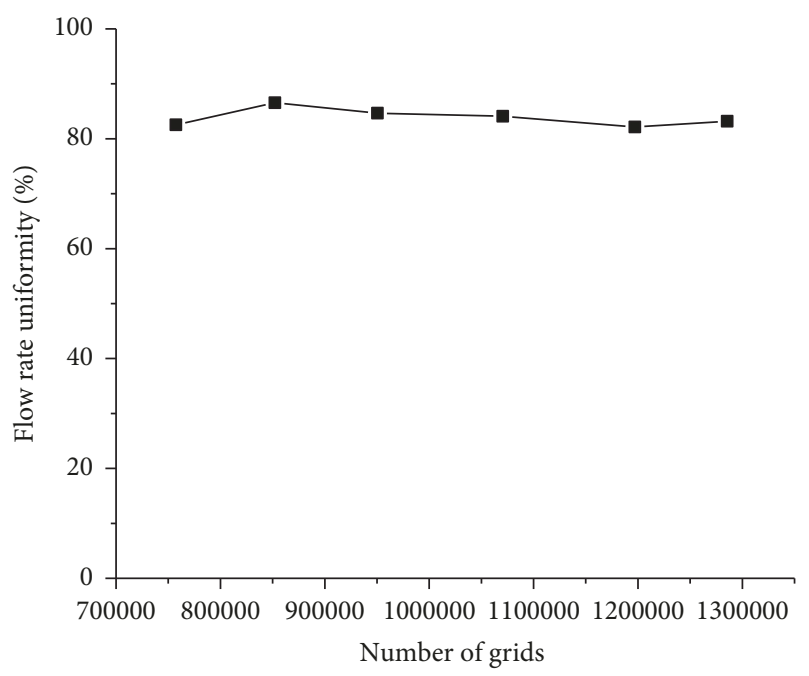

(b) Flow velocity uniformity for different grid numbers

Figure 11

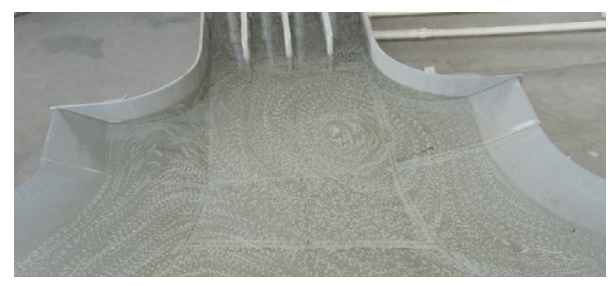

FIGURE 12: Surface flow tracer.

Original Plan. This is a scheme of no rectification measures in the forebay. Due to the lateral flow of the estuary, there are two wide ranges of vortices in the lower left and upper right sides. The two vortices are gradually reduced from the surface to the bottom, and the velocity of the vortex is almost 0 . Forebay will form the deposition after a long period of operation, thus affecting the safety and stability of the operation of the pump station (Figures 13(a) and 13(b)).

"Y" Type Diversion Pier. The "Y" type diversion pier was rectified in the front tank and is arranged symmetrically in the front tank, and its center is located on the G line. The main whirlpool is still concentrated in the lower left corner and the upper right corner of Figures 14(a) and 14(b), and by the surface layer of the fluid, it can be seen on the top right of the large whirlpool edge that a small whirlpool appears. Compared with the original situation, the whirlpool area on the left front slope of the inlet basin is obviously reduced, but the whirlpool is more on the rear side of the diversion pier. The flow pattern is very bad. It is easy to cause siltation here. The bottom streamline bias phenomenon is quite serious; that is, the inlet flow line and the inlet channel direction angle are too large. Analyzing the reasons for this, the " $Y$ " type diversion pier distance from the inlet channel is too small. Rectification measures and the inlet channel are too close, making the flow suddenly change.

"T" Type Diversion Pier. This program uses a "T" type diversion pier for rectification; "T" type diversion pier is in the "Y" type of diversion pier on the basis of adjustment of the angle and position; adjusting the angle is mainly done to make the diversion effect more obvious. Water flows into the left side of the larger proportion, thus eliminating the left side of the whirlpool, which is more effective, while the left edge separates a small whirlpool. In addition, the diversion piers on the back side of the water are also adjusted to reduce the water whirlpool formed on the backwash side after the diversion of the water flow. At the same time, the distance between the diversion pier and the inlet is prolonged, so as to reduce the influence of the whirlpool on the inlet. As can be seen from the surface streamline (Figures 15(a) and 15(b)), the main whirlpool is still concentrated in the lower left and upper right; the bottom streamline and " $Y$ " diversion pier measures compared to the mainstream bias angle have been reduced. The basic flow of water can be smoothly added to the various inlet channels.

Narrow Bottom. The program in the forebay set a height of $4 \mathrm{~cm}$ and a width of $2 \mathrm{~cm}$ for the narrow bottom. The whirlpool on the left has been basically eliminated, while the upper right corner of the whirlpool still exists, and the number increased to 2-3 (Figures 16(a) and 16(b)). Surface water flow can be smoothly added to the inlet channel. As water is blocked by the narrow bottom, the surface layer will exhibit a hammed water phenomenon. After the flow of water, the mainstream area and the surrounding water show turbulence diffusion, so as to achieve the energy of the interaction. Therefore, when flowing into the inlet channel, the flow state changes quite smoothly.

High and Wide Bottom. The program has a high and wide bottom in the forebay to rectify; compared with the narrow bottom, the height and width of the hole have increased, and the rear of the ramp is a slope, which can make the water flow forward over the ridge more smoothly. Surface water flow 

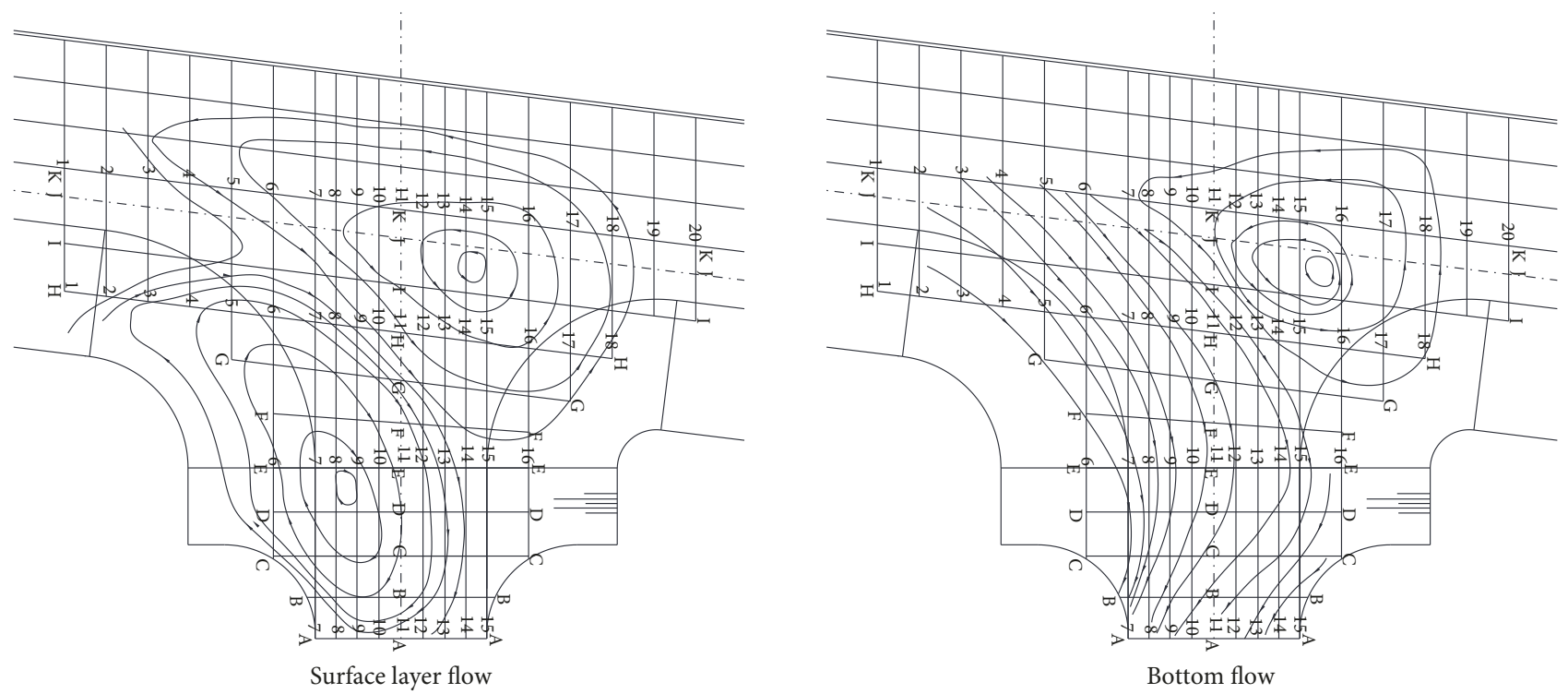

(a) Model test of streamlines
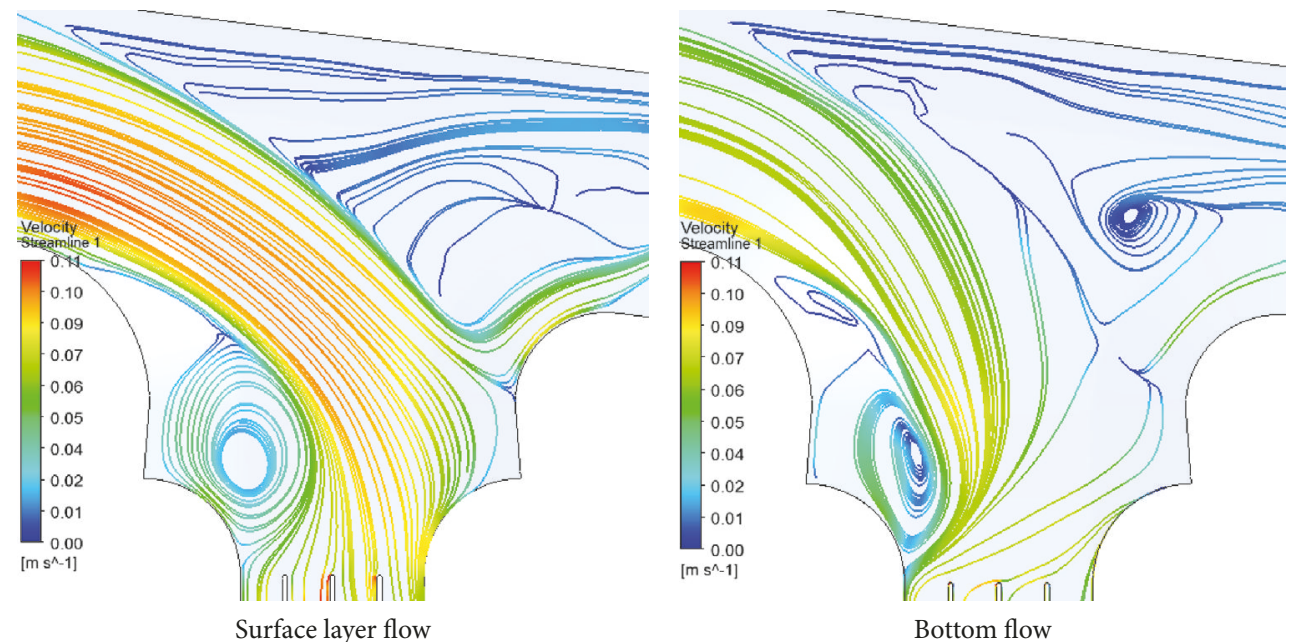

(b) Numerical simulation of streamlines

Figure 13

lines are able to flow smoothly into the pool. In the upper right position, there is still a wide range of whirlpools separated from a small range of whirlpools, but in the postflow, they are better. The bottom of the flow is becoming more ideal; after the ridge has also basically disappeared, the water flows more directly into the pool. As the elevated height of the narrow bottom reduces the overflow area, thereby increasing the flow rate, the back bottom of the swirling almost disappeared. Due to the water blocking of the narrow bottom, the upper right side of the whirlpool range becomes greater and easily causes deposition in the whirlpool central area (Figures 17(a) and 17(b)).

Diversion Wall. The program in the forebay is equipped with a long straight wall for rectification. The main stream flows through the diversion wall and the wall after the poor flow of water convection and energy exchange, making the bottom of the flow gradually tend to smooth. As with the diversion wall distance from the inlet pool, the inlet of the bottom flow has been significantly improved, and the water flows directly into the inlet pool. Smaller whirlpools formed behind the diversion wall, which disappeared before entering the inlet pool (Figures 18(a) and 18(b)).

From Figures 13 to 18 , it can be seen that numerical simulation of the top and bottom flow fields can reproduce the results of the model test.

\subsection{Contrastive Analysis of Model Test and \\ Numerical Simulation of Flow Rate Distribution}

3.2.1. Analysis of Model Test and Numerical Simulation of Flow Rate Distribution. As we can see in Figure 19, the comparative analyses of the velocity distribution of the model test and the numerical simulation are roughly the same. The 

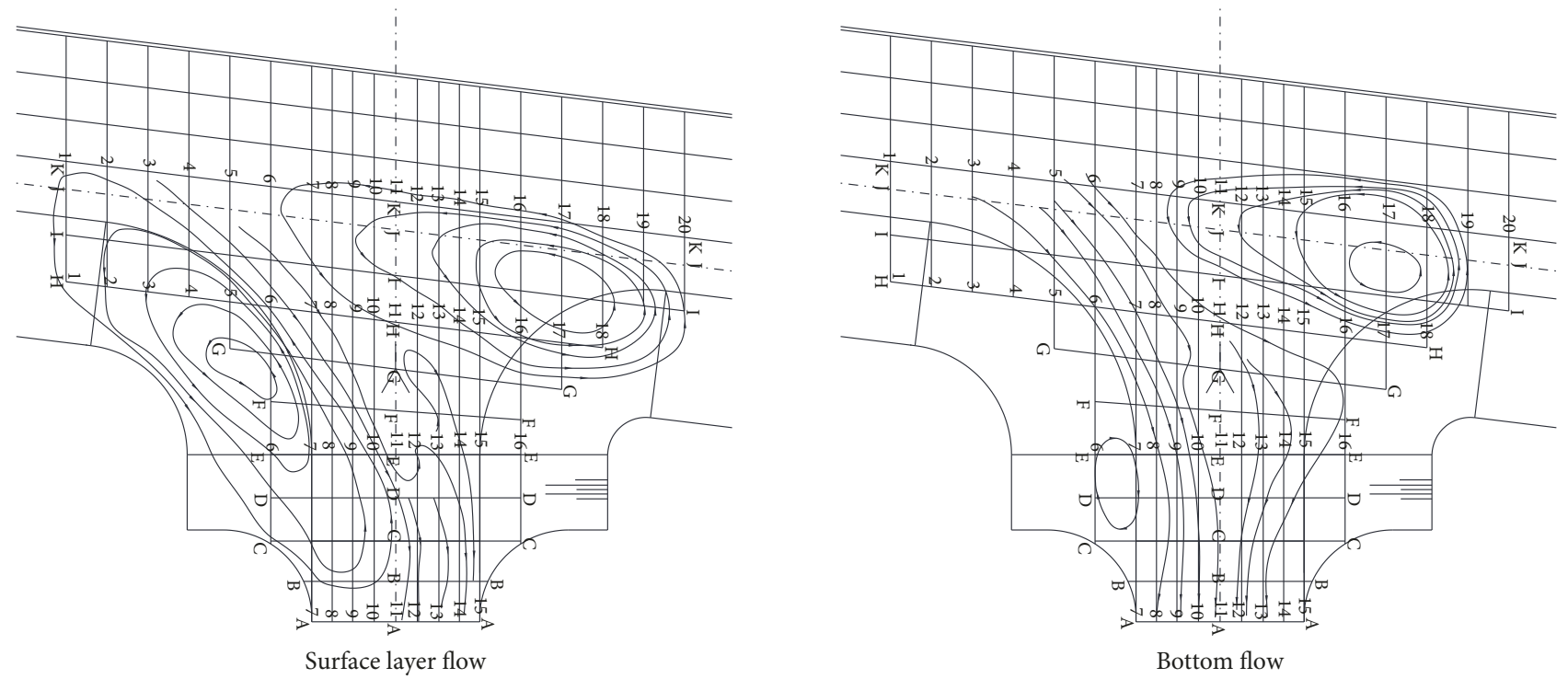

(a) Model test of streamlines
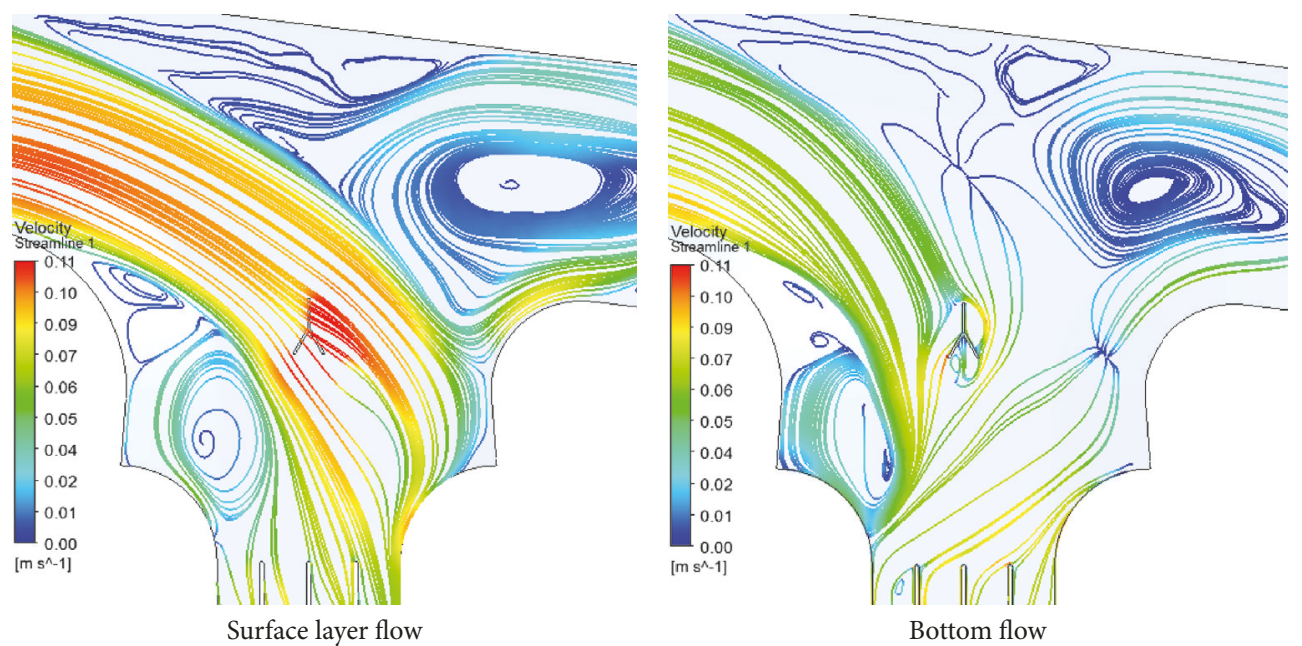

(b) Numerical simulation of streamlines

FIGURE 14

main flow is biased to the right, the right side of the flow rate is generally larger than the left side, and the flow rate from the surface layer to the bottom is gradually reduced. The difference lies in the velocity of the whirlpool on the left side, since the model test does not measure the velocity of the bank slope and its vertical line. There is a large whirlpool on the right side after the lateral water inlet, and with the increase of the water level, the scope of the whirlpool also increases. Because the mainstream tends to the left, it is important to add the rectification measures.

Water flows through the "Y" shaped rectifier, and the rectification effect is better, as the right whirlpool is also greatly reduced. The comparative analyses of the velocity distribution of the model test and the numerical simulation are roughly the same. After the diversion, the main body of water flows to both sides, and the flow velocity from the surface layer to the bottom is gradually reduced, when reaching the inlet position becomes relatively uniform (Figure 20). When entering the inlet channel, A-B cross section flow is relatively uniform. As can be seen from the E section in the figure, there is a swirl in the flow field of the " $Y$ " shaped diversion pier, and the water in the E section appears to roll. But the water flows closer to the inlet, and the flow also tends to be stable; with the water level increases, the flow distribution is more uniform.

The velocity distribution of the section is similar to that of scheme "Y" type diversion pier. As a result of the adjustment of the " $\mathrm{T}$ " type diversion pier and the inlet channel distance, diversion of water can quickly be achieved. The whirlpool after the diversion pier can also be dissipated by the two streams' diversion flow (Figure 21).

The comparative analyses of the velocity distribution of the model test and the numerical simulation are roughly the same. The high velocity area is above the narrow bottom, and the flow velocity on both sides is relatively high. Numerical 

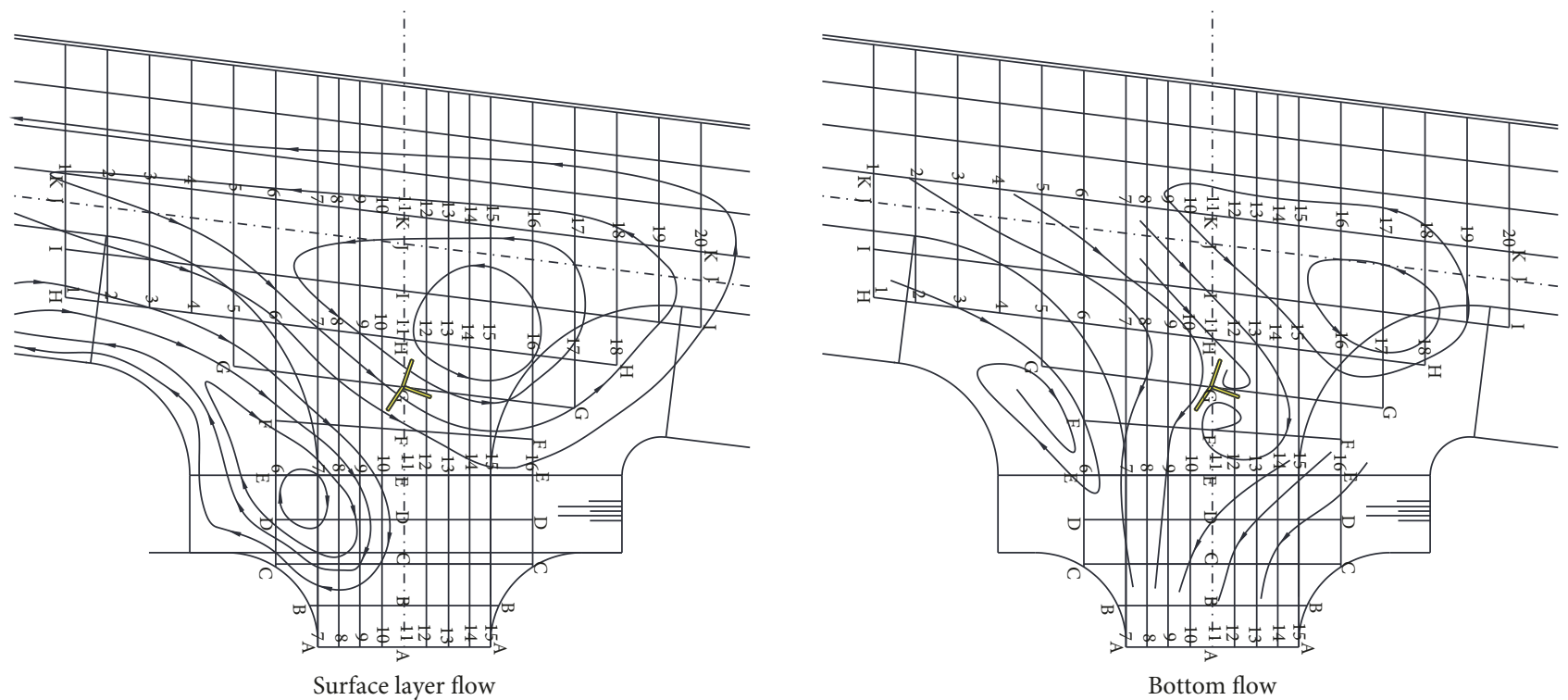

(a) Model test of streamlines
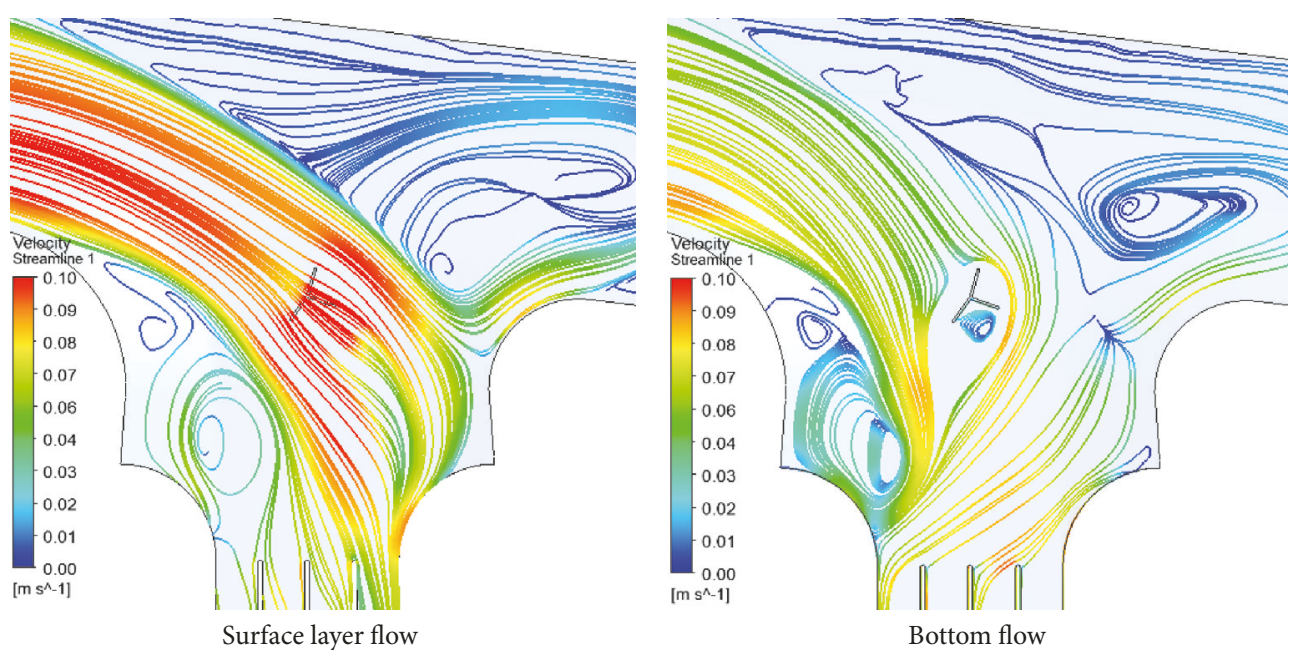

(b) Numerical simulation of streamlines

FIGURE 15

simulation in the narrow bottom will produce a roll; the flow rate will be negative. In the model test, when the flow velocity of the bottom water flow is measured, the flow pattern is chaotic and the flow direction changes quickly, and it is difficult to achieve a relatively stable state. Therefore, the two flow rate distributions have certain differences. After the diversion, the flow velocity from the surface layer to the bottom is gradually reduced, when reaching the inlet position becomes relatively uniform. After the water flows through the narrow bottom, when the water reaches the A-B section, the cross section flow rate is quite uniform. But at both sides of the forebay, due to the curved wing wall contraction, both sides of the velocity exhibit a little change (Figure 22).

The velocity distribution of the section is similar to that of scheme narrow bottom. Because of the slope set on the bottom, rotating water will be controlled. Therefore, the crosssectional flow from $\mathrm{E}$ to $\mathrm{C}$ to $\mathrm{A}-\mathrm{B}$ also achieves uniform flow faster. The main body of water in the E section is in the right side of the surface; with the flow of water forward, the mainstream tends to the left and eventually to the pumping station (Figure 23).

After diversion of the diversion wall, the water flow is mainly distributed to both sides. Both velocity profiles (Figure 24) can be seen; the left side of the flow rate is greater than the right side of the water flow rate. Since there is no measure to reduce the whirlpool after a single diversion wall, this whirlpool has a greater impact on the velocity distribution and even has an impact on velocity distribution of the A-B section. The mainstream is biased towards the right side of the inlet, and there is also a small part of the inflow close to the right pier, making the inlet flow uneven, affecting the pumping station unit efficiency.

From Figures 19-24, it can be seen that the numerical simulation of the cross-sectional flow rate can reproduce the results of the model test. 


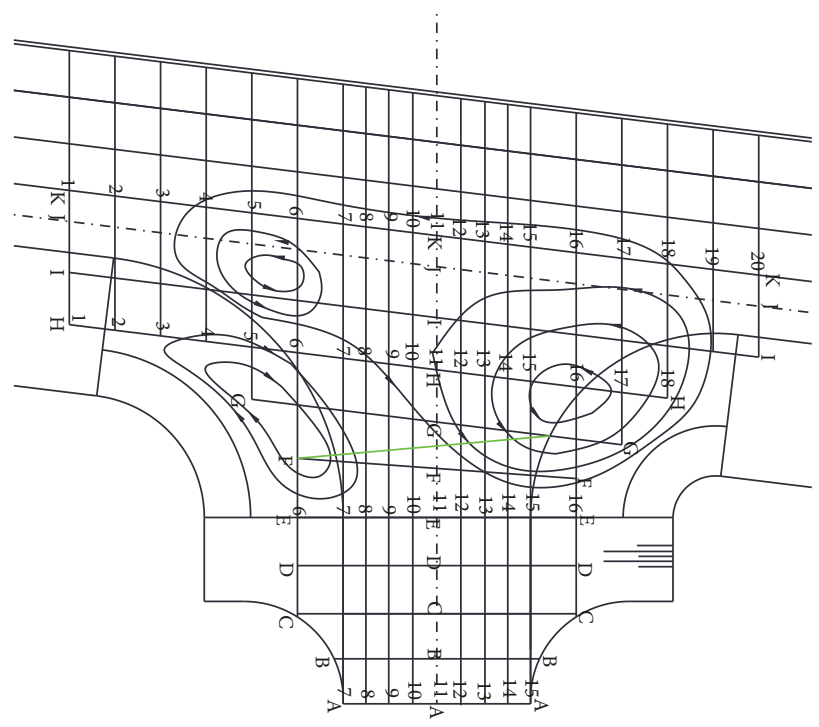

Surface layer flow

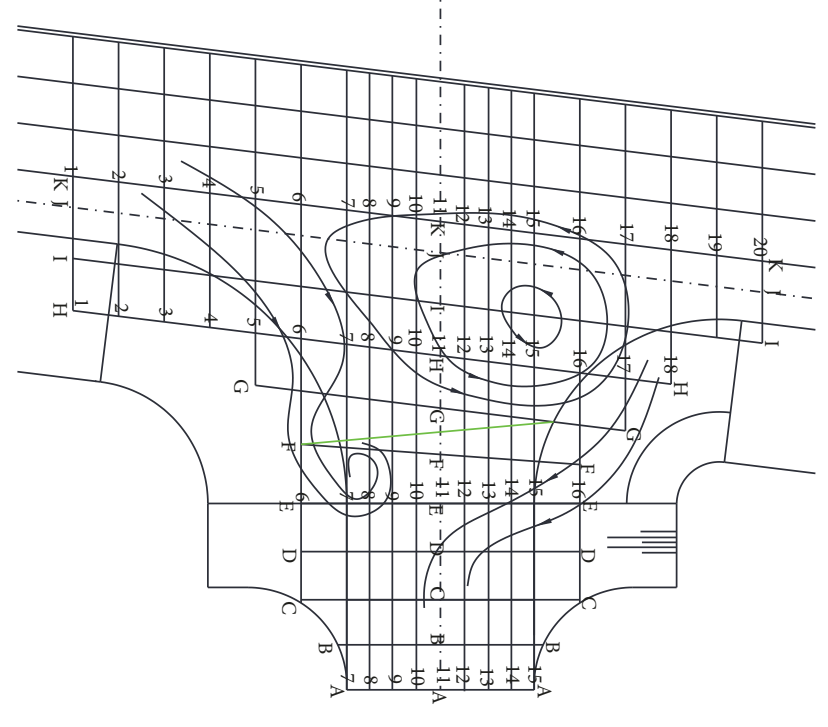

Bottom flow

(a) Model test of streamlines
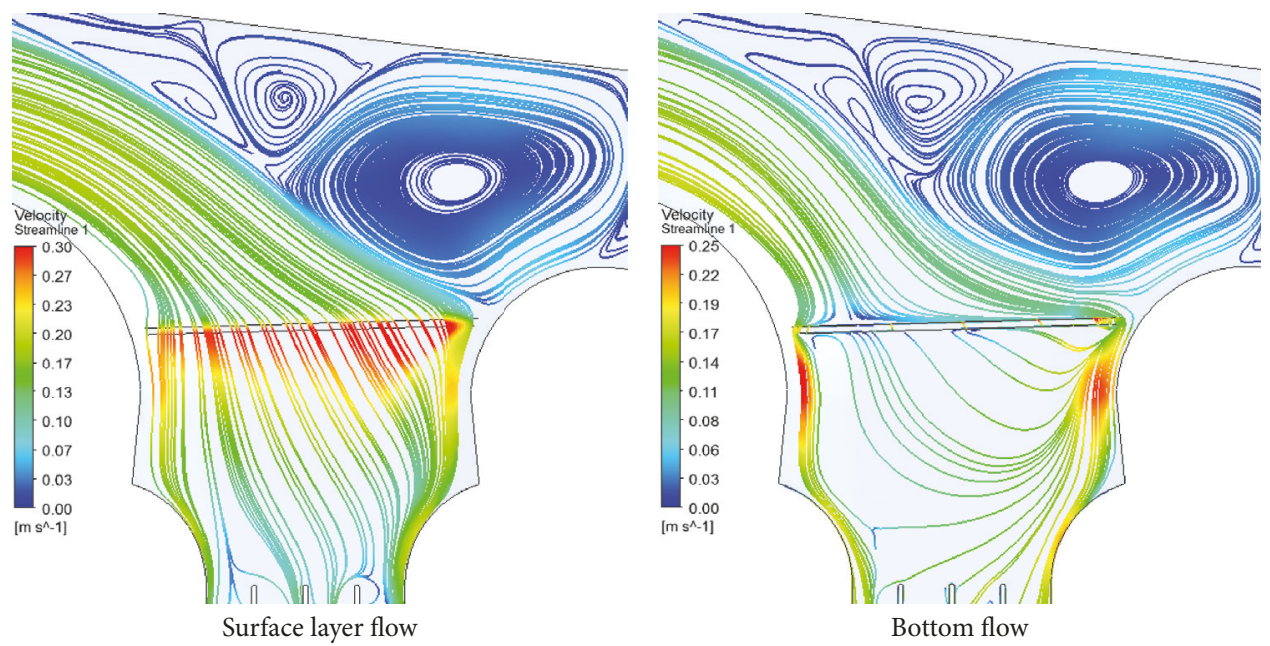

(b) Numerical simulation of streamlines

FIGURE 16

3.2.2. Analysis of Uniformity of Flow Rate Distribution in Model Test and Numerical Simulation. The flow rate distribution uniformity calculation was carried out for the three sections of $\mathrm{A}-\mathrm{B}, \mathrm{C}$, and $\mathrm{E}$ before the inlet. follows:

The velocity distribution uniformity is calculated as

$$
V_{u}=\left[1-\frac{1}{\bar{u}_{a}} \sqrt{\frac{\sum\left(u_{a i}-\bar{u}_{a}\right)^{2}}{m}}\right] \times 100 \%,
$$

where $\bar{u}_{a}$ is the average velocity of the section, $\mathrm{m} / \mathrm{s} ; u_{a i}$ is the flow rate of the $i$ th measuring point, $\mathrm{m} / \mathrm{s} ; m$ is the total number of cross section measuring points.

The results of the flow velocity uniformity are shown in Table 4. The results show that the velocity distribution uniformity of the six schemes is consistent with the observed results. The trend of velocity uniformity change is basically the same as that of the model test and numerical simulation. The closer to the inlet, the more uniform the flow velocity distribution. Comparing the flow velocity uniformity in every scheme, you will find that the flow velocity distribution of scheme 3 and scheme 4 is higher, which is relatively uniform in all schemes.

As can be seen from Figure 25, the results of numerical simulation of each section of scheme 3 are in good agreement with the model tests as compared to other schemes.

\section{Conclusion}

In the absence of any engineering measures (Figures 13 and 19), the flow velocity of the forebay of the pumping station is large. There is a large area of swirling flow in the inner 

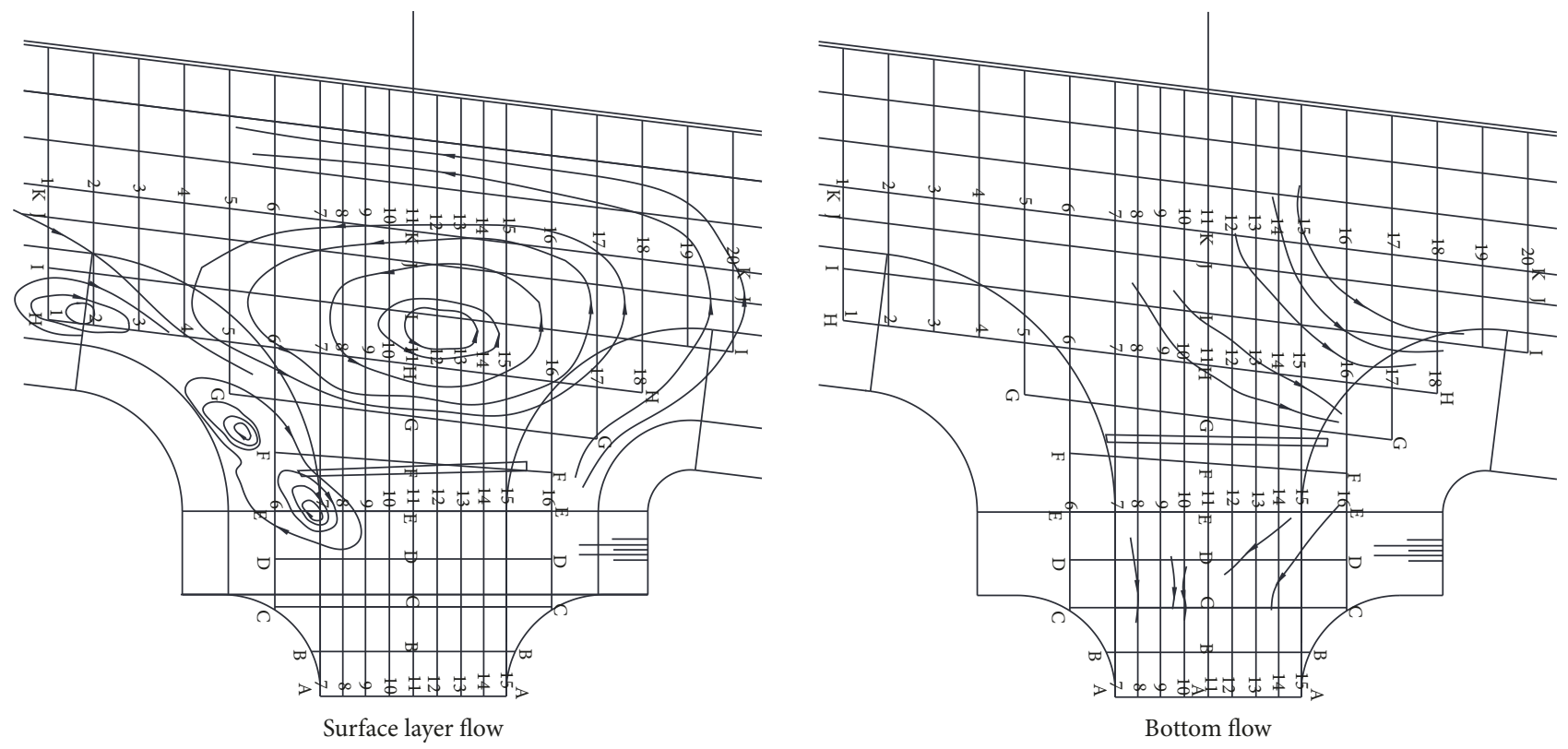

(a) Model test of streamlines
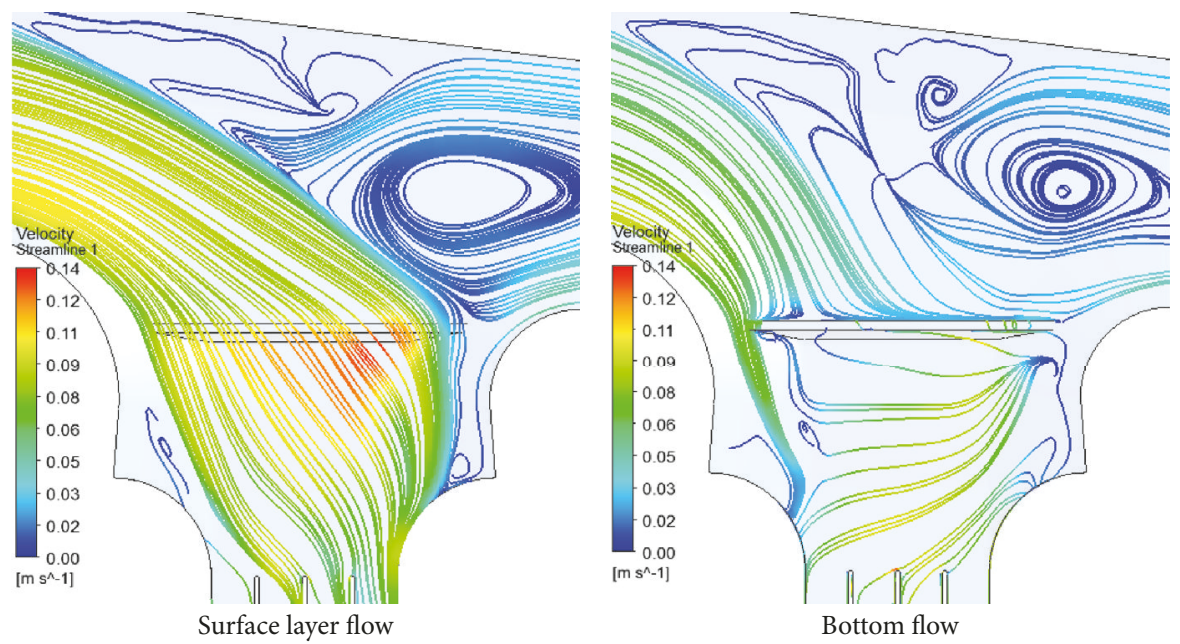

(b) Numerical simulation of streamlines

FIgURE 17

TABLE 4: Section velocity uniformity.

\begin{tabular}{|c|c|c|c|c|}
\hline \multirow{2}{*}{ Program number } & \multirow{2}{*}{ Condition } & \multicolumn{3}{|c|}{ Velocity distribution uniformity $\mathrm{Vu}(\%)$} \\
\hline & & $A-B$ & C & $\mathrm{E}$ \\
\hline \multirow{2}{*}{1} & Model test & 82.13 & 62.26 & 57.18 \\
\hline & Numerical simulation & 77.91 & 61.63 & 55.28 \\
\hline \multirow{2}{*}{2} & Model test & 85.27 & 65.35 & 59.90 \\
\hline & Numerical simulation & 79.08 & 59.04 & 52.51 \\
\hline \multirow{2}{*}{3} & Model test & 82.82 & 67.58 & 62.33 \\
\hline & Numerical simulation & 79.06 & 64.91 & 59.02 \\
\hline \multirow{2}{*}{4} & Model test & 90.56 & 81.32 & 54.26 \\
\hline & Numerical simulation & 73.54 & 54.8 & 39.34 \\
\hline \multirow{2}{*}{5} & Model test & 87.75 & 80.49 & 62.57 \\
\hline & Numerical simulation & 77.38 & 51.36 & 42.88 \\
\hline \multirow{2}{*}{6} & Model test & 80.92 & 62.35 & 60.84 \\
\hline & Numerical simulation & 73.79 & 63.95 & 59.54 \\
\hline
\end{tabular}



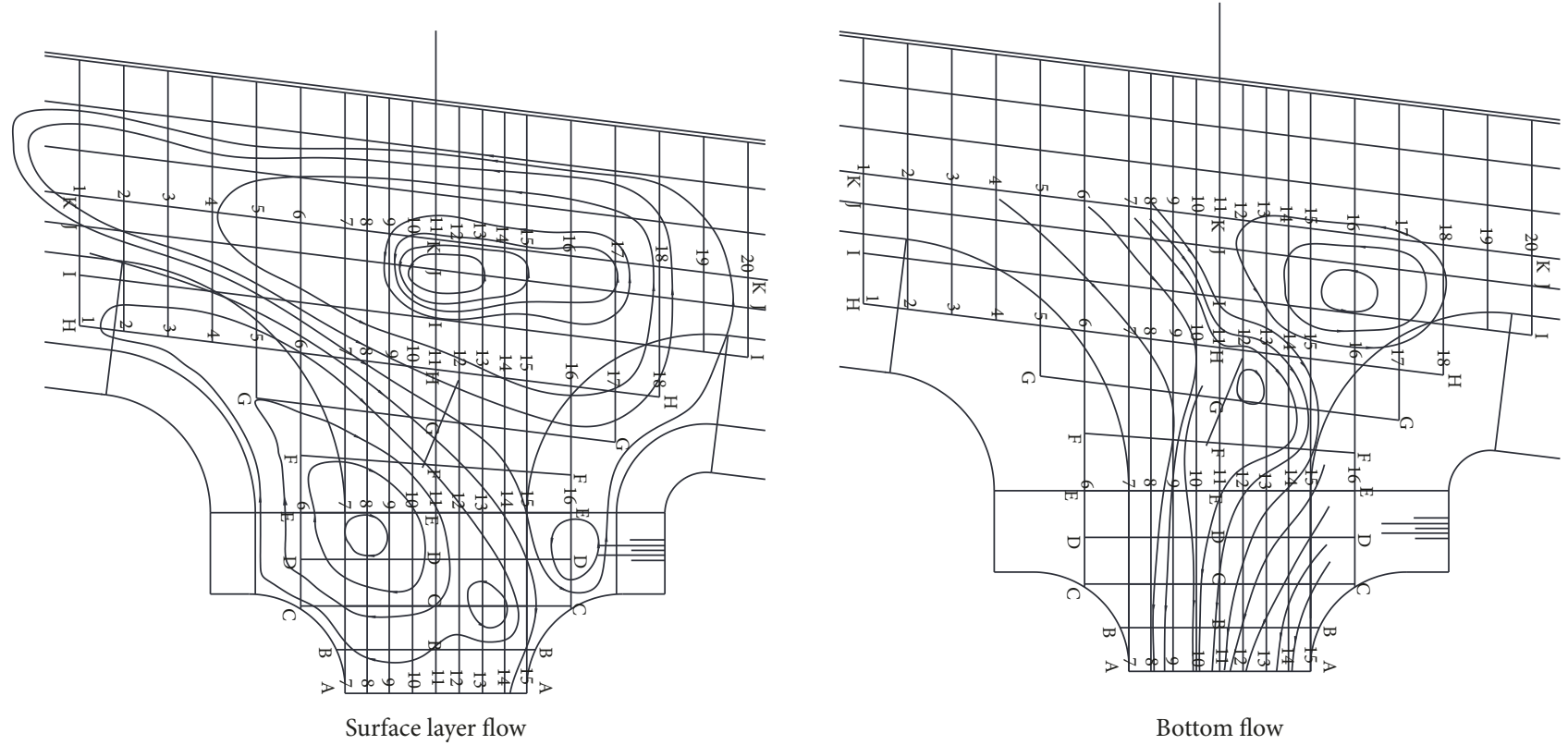

(a) Model test of streamlines

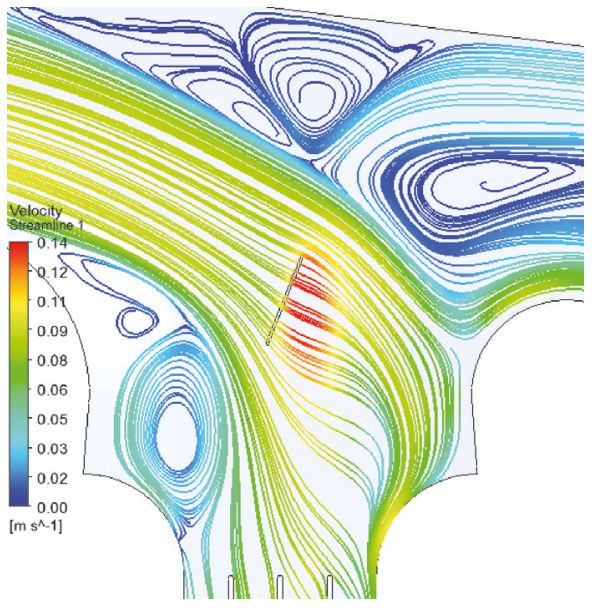

Surface layer flow

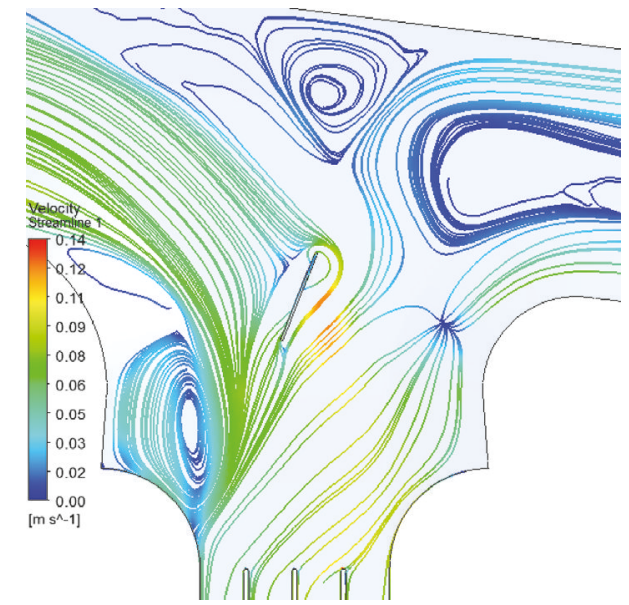

Bottom flow

(b) Numerical simulation of streamlines

Figure 18

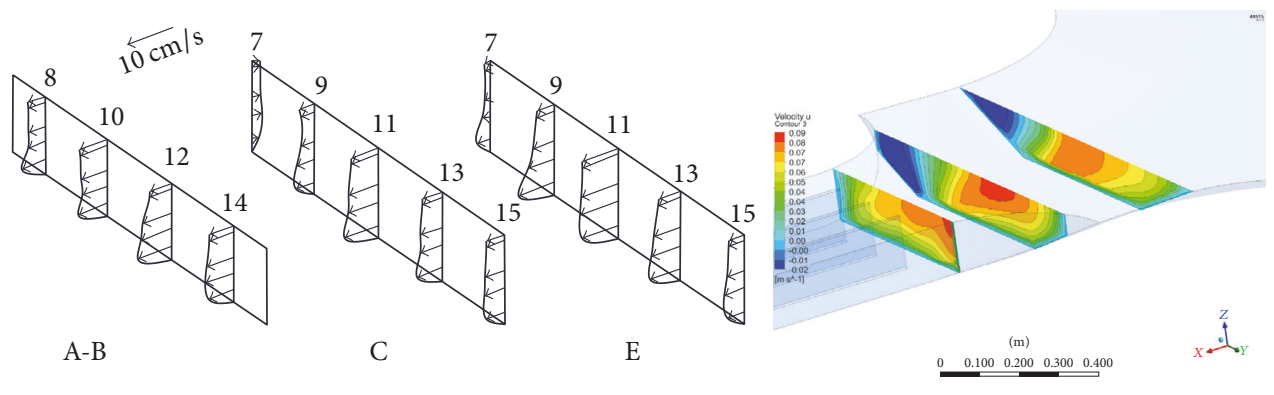

FIGURE 19: Model test and numerical simulation section flow velocity distribution (original plan). 


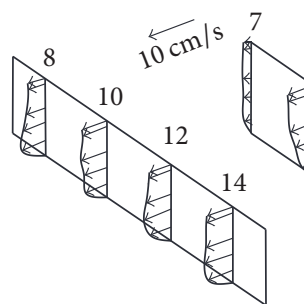

A-B

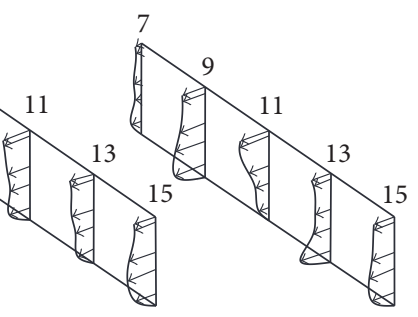

C

E

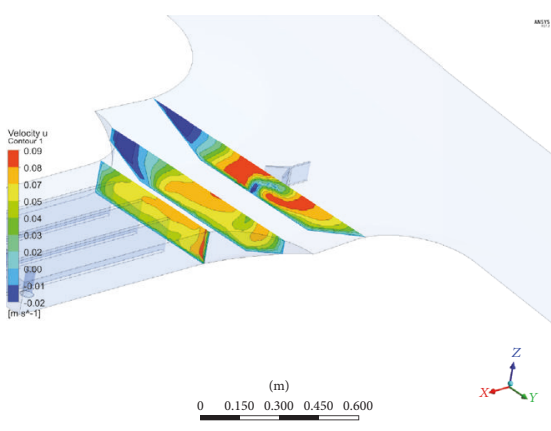

$\underline{0.150 \quad 0.300 \quad 0.450 \quad 0.600}$

FIGURE 20: Model test and numerical simulation section flow velocity distribution ("Y" type diversion pier).

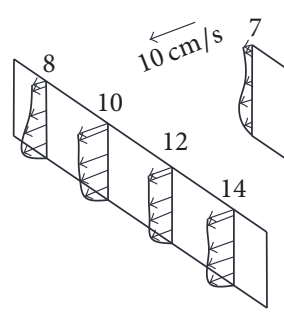

A-B

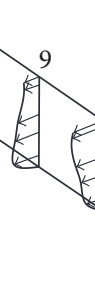

C

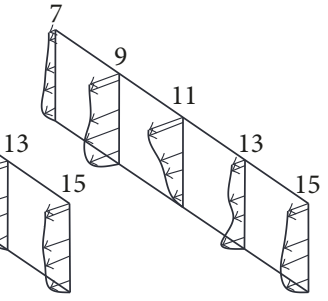

E

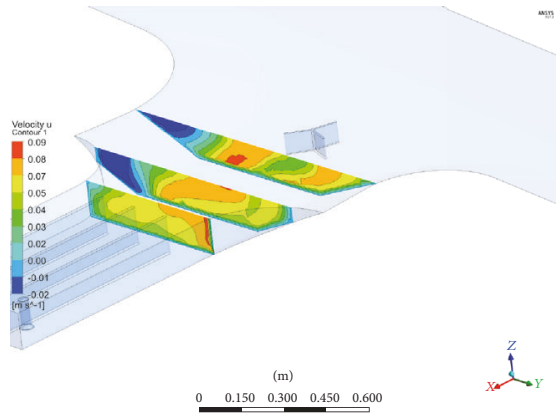

FIGURE 21: Model test and numerical simulation section flow velocity distribution (“T” type diversion pier).

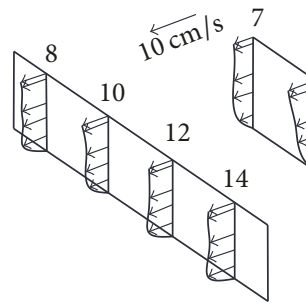

A-B

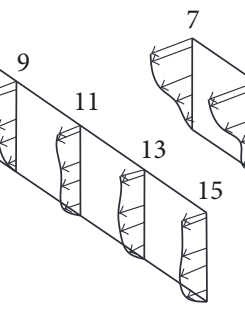

C

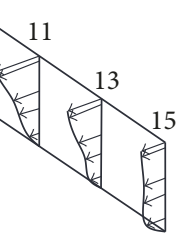

$\mathrm{E}$

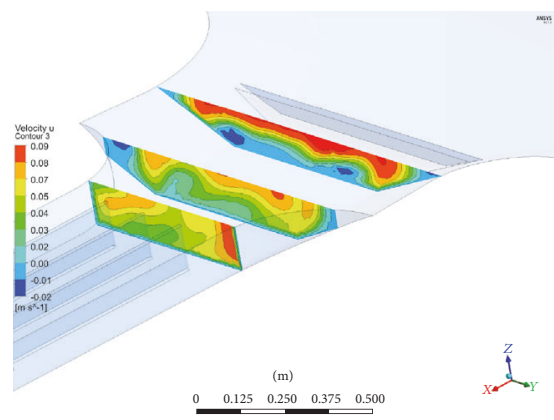

FIGURE 22: Model test and numerical simulation section flow velocity distribution (narrow bottom).

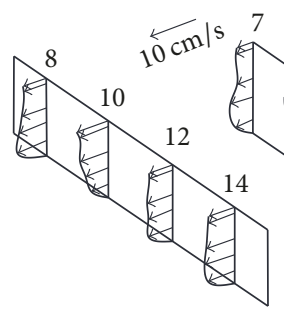

A-B

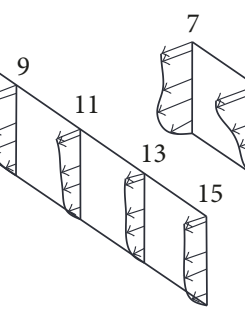

$\mathrm{C}$

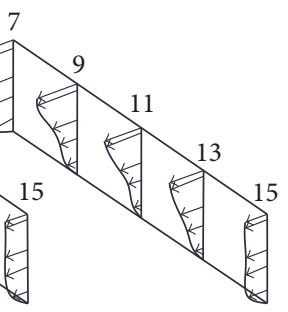

$\mathrm{E}$

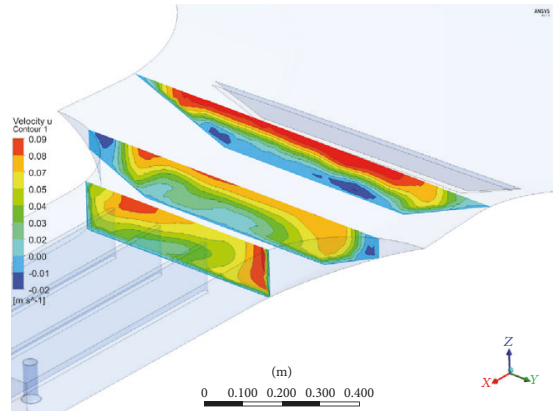

FIGURE 23: Model test and numerical simulation section flow velocity distribution (high and wide bottom).

and bottom layers of the river, which is likely to cause sedimentation in the river. This will increase the influences of the water flow on the safe and economical operation of pumping stations.
In order to optimize the flow pattern of the forebay, five kinds of rectification measures are set up in the front of the pumping station: " $Y$ " type diversion pier, "T" shaped diversion pier, narrow bottom, high and wide bottom, and 


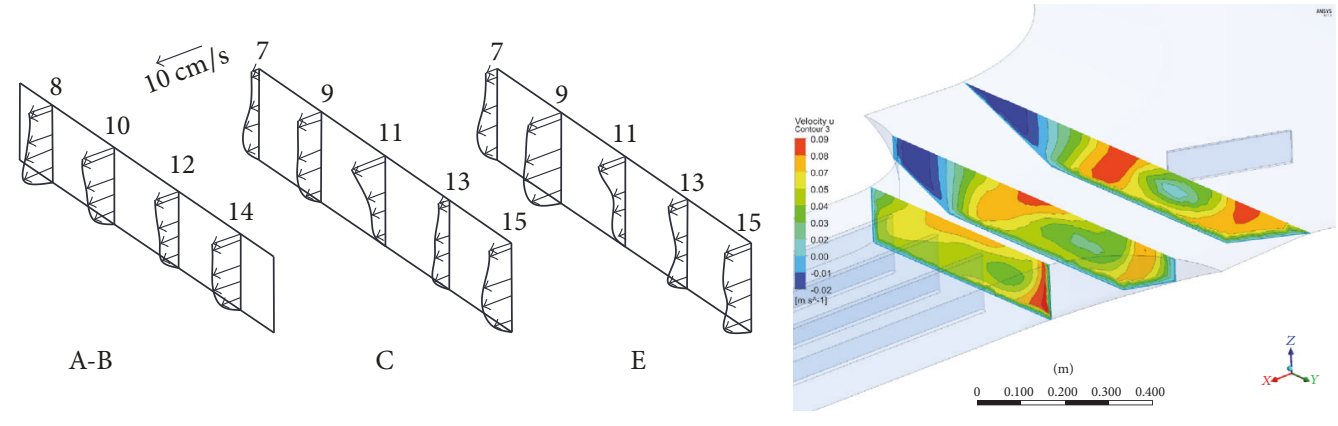

Figure 24: Model test and numerical simulation section flow velocity distribution (diversion wall).

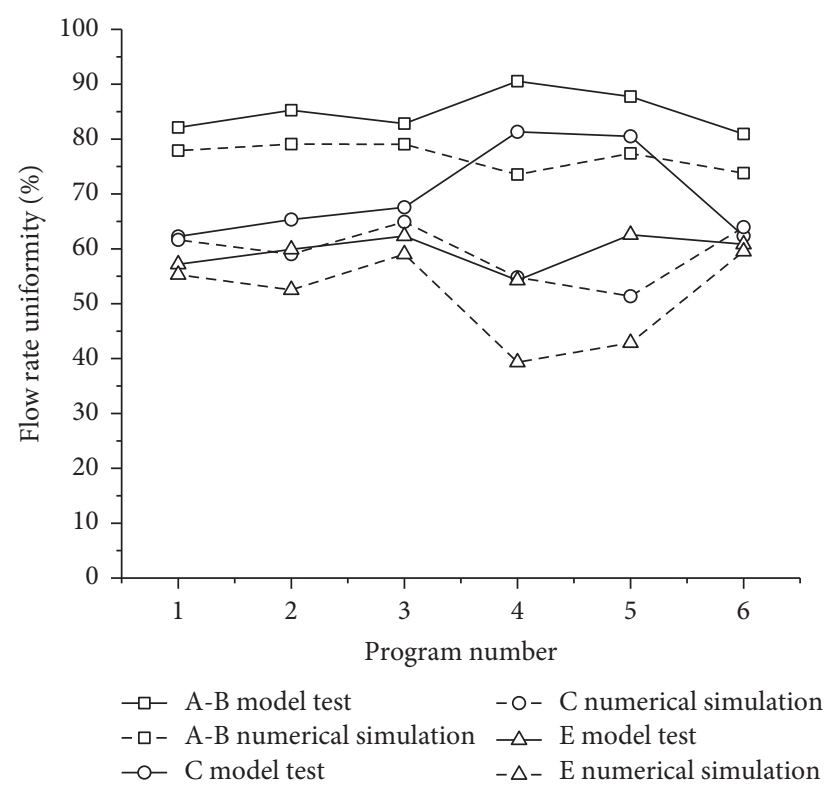

FIGURE 25: Section velocity uniformity.

diversion wall. The surface and bottom flow modes of these five optimization measures were simulated by the model test, and the velocity uniformity of the characteristic cross section was calculated. Finally, the results show that the uniformity of flow and velocity distribution before the station can be improved.

The change of the position, the range, and the number of the vortices in the bottom layer of the scheme and the uniformity of the velocity distribution of each section of the pump foreground are comprehensively compared with each other. Program 3 (" $T$ " shaped diversion pier) is recommended as the pumping station flow control measures. Due to the positive water blocking effect of the " $T$ " shaped diversion piers, the water flow will be separated from the sides of the diversion piers, leading to a sudden change in the flow direction on the left side, so that the vortex range on the left side becomes smaller. The diversion pier can effectively reduce the shunt after the resulting vortex, so as to achieve uniform flow of water into the pumping station. The improvement of water flow in the pumping station can effectively improve the running performance of the pump, reduce the sedimentation, improve the reliability and economy of the pumping station, reduce the cost, and improve the efficiency.

\section{Conflicts of Interest}

The authors declare that they have no conflicts of interest.

\section{Acknowledgments}

This research was supported by the Project Funded by the Priority Academic Program Development of Jiangsu Higher Education Institutions, the National Water Pollution Control and Treatment Science and Technology Major Project (Grant no. 2014ZX07405-002), and Postgraduate Research \& Practice Innovation Program of Jiangsu Province (Grant no. KYCX17_0417). The authors would like to thank the Nanjing Environmental Protection Bureau, Nanjing, for providing monitoring data and associated information.

\section{References}

[1] J. Zhou, C. Liu, F. Tang et al., "Study on improving flow pattern of the complicated forebay of pumping station," Journal of Jiangsu Agricultural College, vol. 19, no. 4, pp. 93-96, 1998.

[2] L. Cheng, L. Chao, Z. Jiren et al., "Discussion on the Side-direction Flow of Pumping Station and Improving of the Flow Pattern," Drainage and Irrigation Machinery, vol. 19, no. 1, pp. 31-34, 2001.

[3] J. Feng and J. Li, "Flow patterns in forebays of large urban water source pumping stations and countermeasures," Advances in Science and Technology of Water Resources, vol. 30, no. 2, pp. 70-74, 2010.

[4] W. Lian, Z. Ma, G. Zhang et al., "Experimental Study on Flow Hydraulic Characteristics of Side Pouring Forebay of Power Plant," China Rural Water and Hydropower, vol. 6, pp. 115-117, 2007.

[5] X. Wang and F. Guo, "Experimental Research on the Hydraulic Characteristics of the Pumping Station's Forbay," Water Conservancy Science and Technology, vol. 09, pp. 597-598, 2006.

[6] L. Cheng, W. Qi, C. Luo, Y. Shang, and H. Yuan, "Effect of geometric parameters of Y-shaped diversion piers on flow pattern in forebay of pumping station," Advances in Science and Technology of Water Resources, vol. 34, no. 1, pp. 68-72, 2014.

[7] C. Luo, L. Cheng, and C. Liu, "Numerical simulation of mechanism for sill rectifying flow in pumping station intake," Paiguan 
Jixie Gongcheng Xuebao/Journal of Drainage and Irrigation Machinery Engineering, vol. 32, no. 5, pp. 393-398, 2014.

[8] C. Ying, Three - Dimensional Numerical Simulation Study on Flow Pattern Improvement of Pumping Station, Yangzhou University, 2007.

[9] J. Huang and W. Liu, "Numerical simulation of flow field in front of pumping station," Science \& Technology Information, vol. 09, pp. 770-771, 2009.

[10] M. Ansar and T. Nakato, "Experimental study of 3D pump-intake flows with and without cross flow," Journal of Hydraulic Engineering, vol. 127, no. 10, pp. 825-834, 2001.

[11] V. P. Rajendran, S. G. Constantinescu, and V. C. Patel, "Experimental validation of numerical model of flow in pump-intake bays," Journal of Hydraulic Engineering, vol. 125, no. 11, pp. 1119$1125,1999$.

[12] V. P. Rajendran and V. C. Patel, "Measurement of vortices in model pump-intake bay by PIV," Journal of Hydraulic Engineering, vol. 126, no. 5, pp. 322-334, 2000.

[13] V. Yakhot and S. A. Orszag, "Renormalization group analysis of turbulence. I. Basic theory," Journal of Scientific Computing, vol. 1, no. 1, pp. 3-51, 1986.

[14] C. G. Speziale and S. Thangam, "Analysis of an RNG based turbulence model for separated flows," International Journal of Engineering Science, vol. 30, no. 10, pp. 1379-1388, 1992.

[15] V. Yakhot, S. A. Orszag, S. Thangam, T. B. Gatski, and C. G. Speziale, "Development of turbulence models for shear flows by a double expansion technique," Physics of Fluids A. Fluid Dynamics, vol. 4, no. 7, pp. 1510-1520, 1992.

[16] H. Zhang, Y. Liang, and X. Zhou, "Sensitivity analysis and optimal operation control for large-scale waterflooding pipeline network of oilfield," Journal of Petroleum Science \& Engineering, 2017.

[17] C. J. Muller and I. K. Craig, "Economic hybrid non-linear model predictive control of a dual circuit induced draft cooling water system," Journal of Process Control, vol. 53, pp. 37-45, 2017.

[18] R. Kernan, X. Liu, S. McLoone, and B. Fox, "Demand side management of an urban water supply using wholesale electricity price," Applied Energy, vol. 189, pp. 395-402, 2017.

[19] F. Wamalwa, S. Sichilalu, and X. Xia, "Optimal control of conventional hydropower plant retrofitted with a cascaded pumpback system powered by an on-site hydrokinetic system," Energy Conversion and Management, vol. 132, pp. 438-451, 2017.

[20] E. M. Wanjiru, L. Zhang, and X. Xia, "Model predictive control strategy of energy-water management in urban households," Applied Energy, vol. 179, pp. 821-831, 2016.

[21] R. Kernan, X. A. Liu, S. McLoone, and B. Fox, "Management of public water supply to reduce energy cost and improve wind power uptake," in Proceedings of the 16th International Conference on Environment and Electrical Engineering, EEEIC 2016, pp. 1-6, June 2016.

[22] C. J. Muller and I. K. Craig, "Energy reduction for a dual circuit cooling water system using advanced regulatory control," Applied Energy, vol. 171, pp. 287-295, 2016.

[23] R. Menke, E. Abraham, P. Parpas, and I. Stoianov, "Demonstrating demand response from water distribution system through pump scheduling," Applied Energy, vol. 170, pp. 377-387, 2016.

[24] B. P. Numbi and X. Xia, "Optimal energy control of a crushing process based on vertical shaft impactor," Applied Energy, vol. 162, pp. 1653-1661, 2016.

[25] P. Olszewski, "Genetic optimization and experimental verification of complex parallel pumping station with centrifugal pumps," Applied Energy, vol. 178, pp. 527-539, 2016.
[26] Z. Zhang, X. He, and A. Kusiak, "Data-driven minimization of pump operating and maintenance cost," Engineering Applications of Artificial Intelligence, vol. 40, pp. 37-46, 2015.

[27] J.-Q. Zhu and Y.-Q. Li, "Computation and simulation analysis of twin-screw pump efficiency under frequency conversion," Advanced Materials Research, vol. 317-319, pp. 2331-2334, 2011.

[28] R. Zhou, J.-J. Li, and Y. Chen, "The optimized white differential equation of $\operatorname{GM}(1,1)$ based on the original grey differential equation," Applied Mechanics and Materials, vol. 166-169, pp. 2971-2975, 2012.

[29] B.-G. Zhan, Y.-D. Ruan, and D. Han, "Determination of ultimate filling height of slag subgrade with FEM modelling," Applied Mechanics and Materials, vol. 188, pp. 84-89, 2012.

[30] A.-M. Georgescu, S. Perju, S.-C. Georgescu, and A. Anton, "Numerical model of a district water distribution system in Bucharest," in Proceedings of the 12th International Conference on Computing and Control for the Water Industry, CCWI 2013, pp. 707-714, September 2013.

[31] A.-M. Georgescu, C.-I. Cosoiu, S. Perju, S.-C. Georgescu, L. Hasegan, and A. Anton, "Estimation of the efficiency for variable speed pumps in EPANET compared with experimental data," in Proceedings of the 16th International Conference on Water Distribution System Analysis, WDSA 2014, vol. 89, pp. 1404-1411, July 2014.

[32] A. Marchi, A. R. Simpson, and N. Ertugrul, "Assessing variable speed pump efficiency in water distribution systems," Drinking Water Engineering and Science, vol. 5, no. 1, pp. 15-21, 2012. 


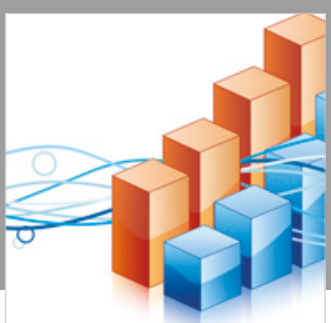

Advances in

Operations Research

\section{-n-m}
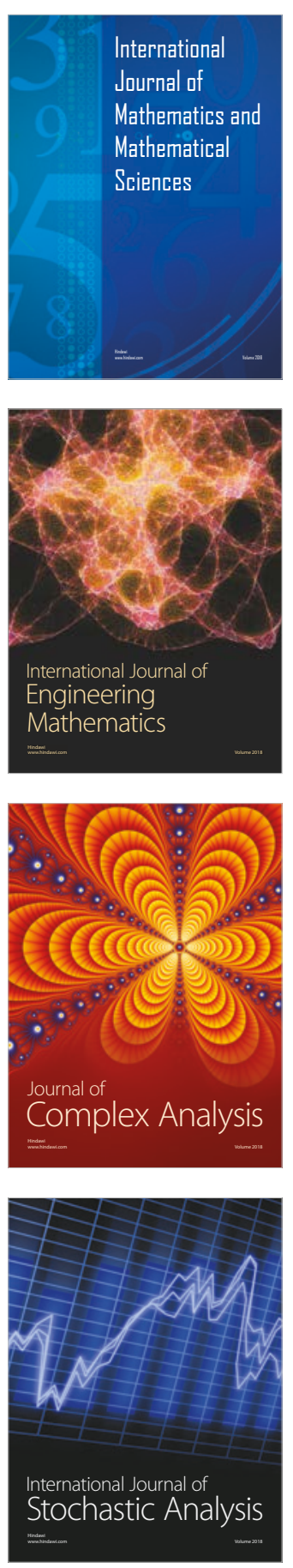
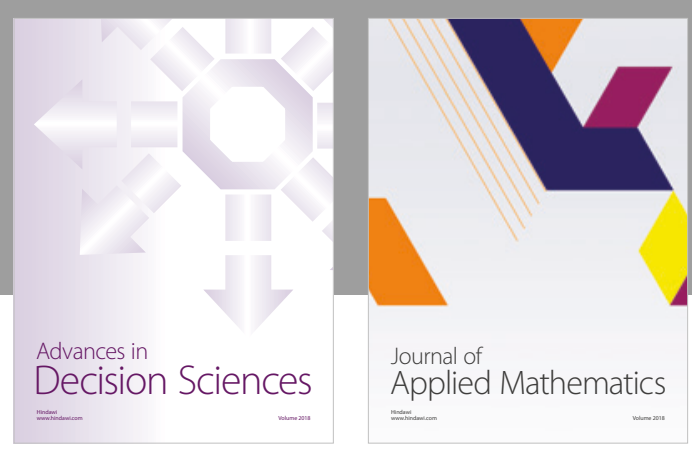

Journal of

Applied Mathematics
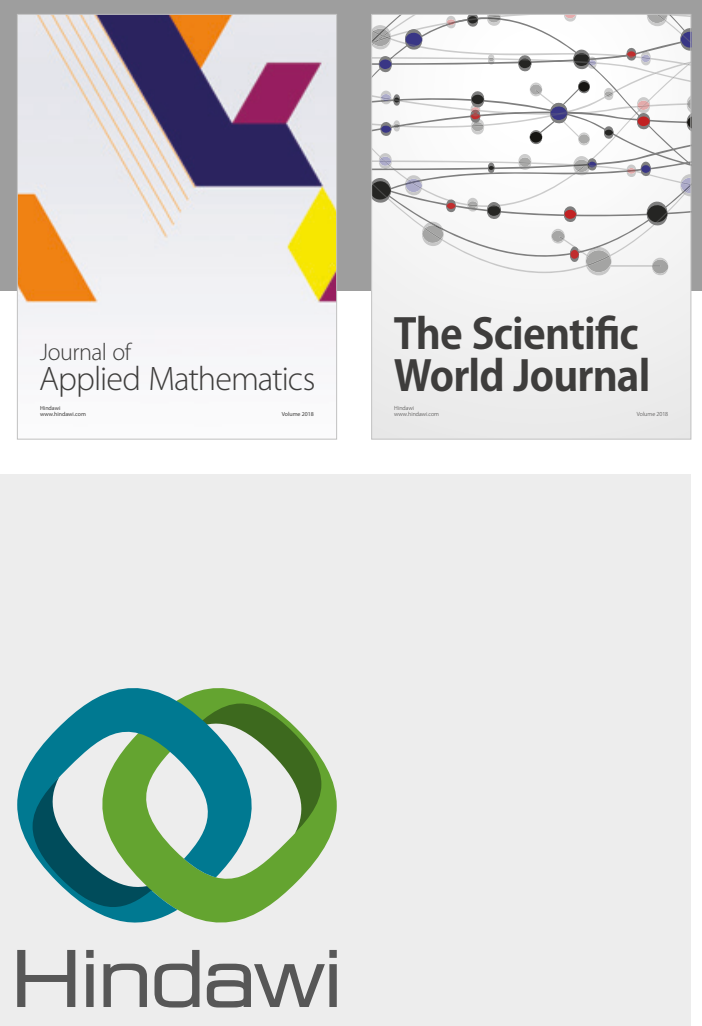

Submit your manuscripts at

www.hindawi.com

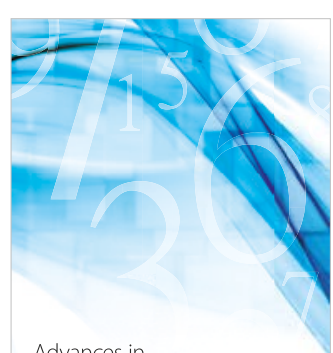

Advances in
Numerical Analysis
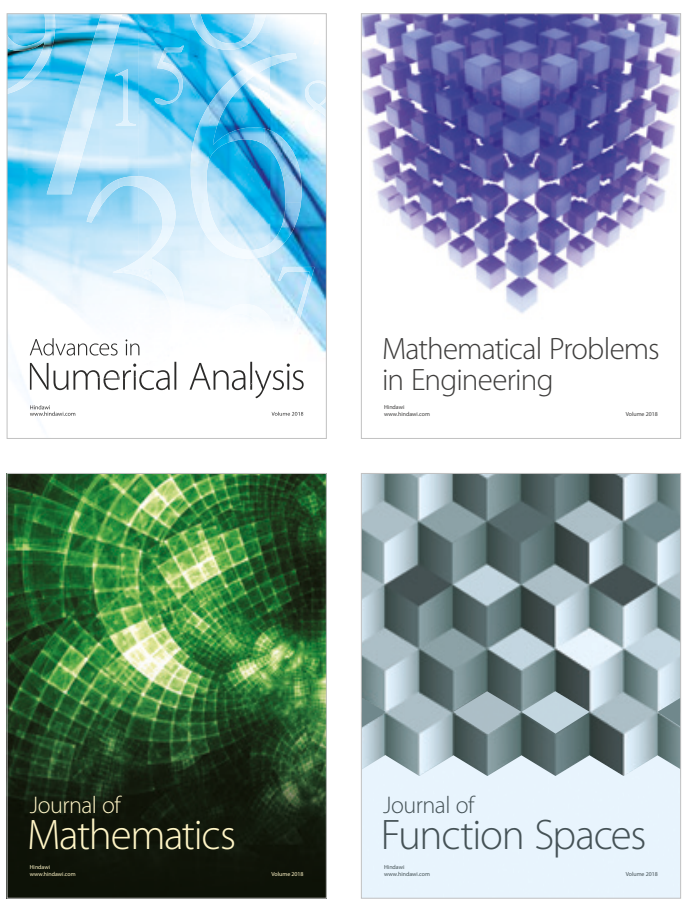

Mathematical Problems in Engineering

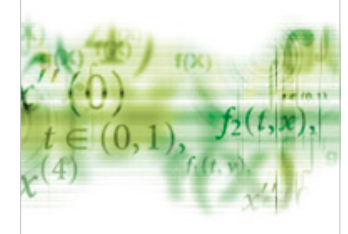

International Journal of

Differential Equations

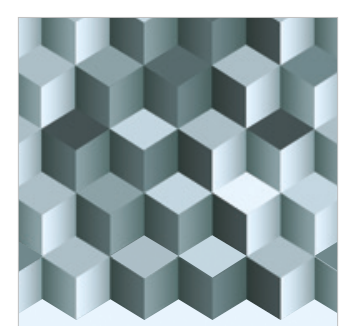

Journal of

Function Spaces

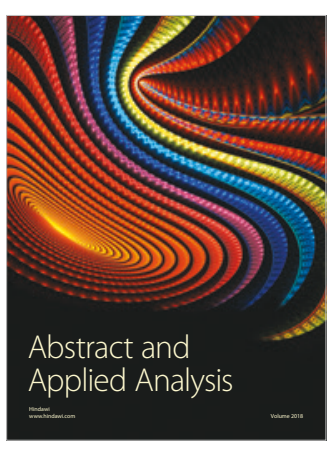

The Scientific

World Journal

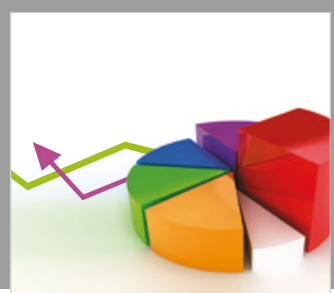

Journal of

Probability and Statistics
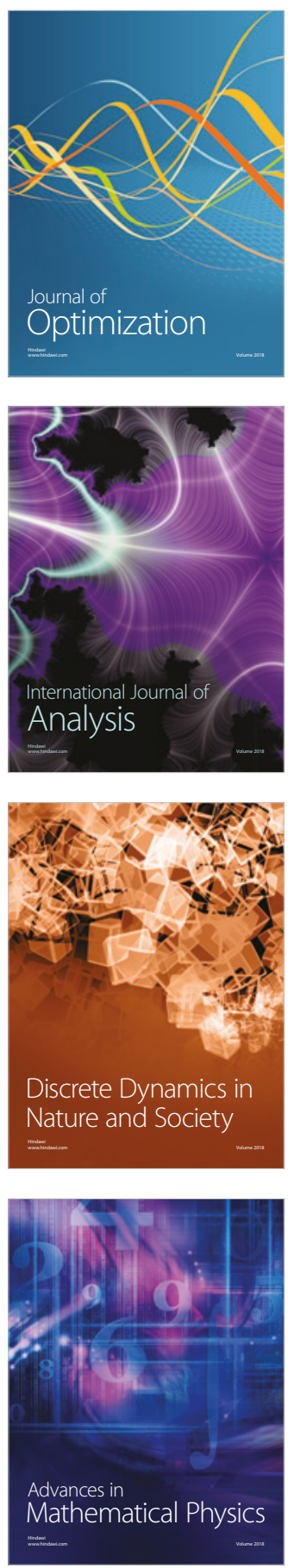